\title{
ON ERROR ESTIMATES OF THE PROJECTION METHODS FOR THE NAVIER-STOKES EQUATIONS: SECOND-ORDER SCHEMES
}

\author{
JIE SHEN
}

\begin{abstract}
We present in this paper a rigorous error analysis of several projection schemes for the approximation of the unsteady incompressible NavierStokes equations. The error analysis is accomplished by interpreting the respective projection schemes as second-order time discretizations of a perturbed system which approximates the Navier-Stokes equations. Numerical results in agreement with the error analysis are also presented.
\end{abstract}

\section{INTRODUCTION}

In this paper, we are concerned with the accuracy of certain projection schemes for the approximation of the Navier-Stokes equations. Let $\Omega \in \mathbb{R}^{d}$ (with $d=2$ or 3 ) be an open bounded set with a sufficiently smooth boundary. We consider the unsteady incompressible Navier-Stokes equations in the primitive variable formulation:

$$
\begin{aligned}
& \boldsymbol{u}_{t}-\nu \Delta \boldsymbol{u}+(\boldsymbol{u} \cdot \nabla) \boldsymbol{u}+\nabla p=\boldsymbol{f} \quad \text { in } \Omega \times[0, T] \\
& \operatorname{div} \boldsymbol{u}=0 \quad \text { in } \Omega \times[0, T],\left.\quad \boldsymbol{u}\right|_{t=0}=\boldsymbol{u}_{0},
\end{aligned}
$$

where the unknowns are the vector function $\boldsymbol{u}$, which represents the velocity of the flow, and the scalar function $p$, which represents the pressure field. The equations (1.1)-(1.2) should be completed with an appropriate boundary condition for the velocity $\boldsymbol{u}$. For the sake of simplicity, we consider the homogeneous Dirichlet boundary condition, i.e., $\left.\boldsymbol{u}\right|_{\partial \Omega}=0, \forall t \in[0, T]$.

One of the main difficulties in solving (1.1)-(1.2) is that the unknowns $(\boldsymbol{u}, p)$ are coupled together by the incompressibility constraint $\nabla \boldsymbol{u}=0$. The projection method, introduced by Chorin $[\mathbf{2}]$ and Temam [20], was designed to overcome this difficulty. Although the projection methods have been widely used because of their efficiency and simplicity (cf. $[\mathbf{1}, \mathbf{5}, \mathbf{6}, \mathbf{1 0}, \mathbf{2 3}]$ and the references therein), a rigorous error analysis for these projection schemes has not been available until recently. In $[\mathbf{1 5}, \mathbf{1 7}]$, the author gave a first error analysis for some frequently used projection schemes. Recently, Rannacher in $[\mathbf{1 2}]$ derived improved optimal first-order error

\footnotetext{
Received by the editor March 3, 1994 and, in revised form, February 11, 1995 and March 6, 1995.

1991 Mathematics Subject Classification. Primary 65M15, 35Q30; Secondary 35A40, 65J15.

Key words and phrases. Pseudo-compressibility, pressure stabilization, projection method, Navier-Stokes equations.

This work was supported in part by NSF Grant \#9205300.
} 
estimates for the original projection scheme introduced in $[\mathbf{2}]$ and $[\mathbf{2 0}]$ (see also $[\mathbf{1 6}]$ and $[4])$.

Consider, for instance, the following projection scheme analyzed in [17]:

$$
\left\{\begin{array}{l}
\frac{\tilde{\boldsymbol{u}}^{n+1}-\boldsymbol{u}^{n}}{k}-\frac{\nu}{2} \Delta\left(\tilde{\boldsymbol{u}}^{n+1}+\boldsymbol{u}^{n}\right)+\tilde{B}\left(\frac{\tilde{\boldsymbol{u}}^{n+1}+\boldsymbol{u}^{n}}{2}, \frac{\tilde{\boldsymbol{u}}^{n+1}+\boldsymbol{u}^{n}}{2}\right)+\nabla p^{n}=\boldsymbol{f}\left(t_{n+\frac{1}{2}}\right) \\
\left.\left(\tilde{\boldsymbol{u}}^{n+1}+\boldsymbol{u}^{n}\right)\right|_{\partial \Omega}=0
\end{array}\right.
$$

$$
\left\{\begin{array}{l}
\frac{\boldsymbol{u}^{n+1}-\tilde{\boldsymbol{u}}^{n+1}}{k}+\frac{1}{2} \nabla\left(p^{n+1}-p^{n}\right)=0 \\
\nabla \boldsymbol{u}^{n+1}=0 \\
\left.\boldsymbol{u}^{n+1} \cdot \boldsymbol{n}\right|_{\partial \Omega}=0
\end{array}\right.
$$

where $k$ is the time step, $t_{n+\frac{1}{2}}=\left(n+\frac{1}{2}\right) k$ and $\tilde{B}(\boldsymbol{u}, \boldsymbol{v})=(\boldsymbol{u} \cdot \nabla) \boldsymbol{v}+\frac{1}{2}(\nabla \boldsymbol{u}) \boldsymbol{v}$ is the modified bilinear form which ensures the dissipativity of the scheme. We note that an implicit treatment for the nonlinear term is used in order to ensure the uniform stability of the semidiscretized system. When the system (1.3)-(1.4) is further discretized in space, it is a common practice to treat the nonlinear term explicitly so long as the discretization parameters satisfy the CFL stability condition.

Let $P$ be the projector in $\boldsymbol{L}^{2}(\Omega)$ onto the divergence-free subspace

$$
\boldsymbol{H}=\left\{\boldsymbol{v} \in \boldsymbol{L}^{2}(\Omega): \nabla \boldsymbol{v} \in L^{2}(\Omega),\left.\boldsymbol{v} \cdot \boldsymbol{n}\right|_{\partial \Omega}=0\right\} .
$$

We infer from (1.4) that $\boldsymbol{u}^{n+1}=P \tilde{\boldsymbol{u}}^{n+1}$, which explains why we call (1.3)-(1.4) a projection scheme.

We note that $\boldsymbol{u}^{n+1}$ can be eliminated from (1.3)-(1.4). In fact, taking the sum of (1.3) at step $n$ and (1.4) at step $n-1$, and applying the divergence operator to (1.4), we obtain

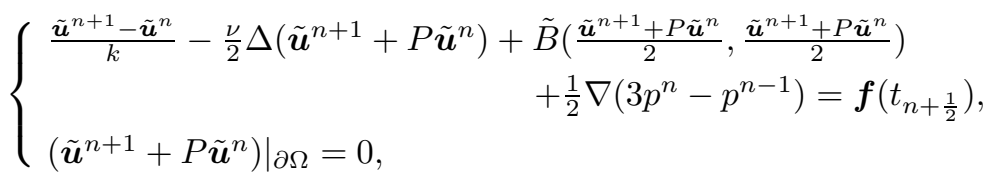

$$
\begin{aligned}
& \nabla \tilde{\boldsymbol{u}}^{n+1}-\frac{1}{2} k \Delta\left(p^{n+1}-p^{n}\right)=0,\left.\frac{\partial p^{n+1}}{\partial \boldsymbol{n}}\right|_{\partial \Omega}=\left.\frac{\partial p^{n}}{\partial \boldsymbol{n}}\right|_{\partial \Omega} .
\end{aligned}
$$

The advantage of reformulating (1.3)-(1.4) is that we can interpret the scheme (1.6)-(1.7) as a second-order time discretization, with a decoupled system for $\left(\tilde{\boldsymbol{u}}^{n+1}, p^{n+1}\right)$, to the perturbed system (see similar interpretations in $[\mathbf{1 8}]$ and $[\mathbf{1 3}]$ ):

$$
\begin{aligned}
& \boldsymbol{u}_{t}^{\varepsilon}-\nu \Delta \boldsymbol{u}^{\varepsilon}+\tilde{B}\left(\boldsymbol{u}^{\varepsilon}, \boldsymbol{u}^{\varepsilon}\right)+\nabla p^{\varepsilon}=\boldsymbol{f},\left.\quad \boldsymbol{u}^{\varepsilon}\right|_{\partial \Omega}=0, \\
& \operatorname{div} \boldsymbol{u}^{\varepsilon}-\varepsilon \Delta p_{t}^{\varepsilon}=0,\left.\quad \frac{\partial p_{t}^{\varepsilon}}{\partial \boldsymbol{n}}\right|_{\partial \Omega}=0,
\end{aligned}
$$

with $\varepsilon \sim \frac{1}{2} k^{2}$. On the other hand, the perturbed system (1.8)-(1.9) can be viewed as an approximation, when $\varepsilon \ll 1$, to the Navier-Stokes equations (1.1)-(1.2). It was thoroughly studied in a recent work $[\mathbf{1 4}]$ in which the following theorem was proved. 
Theorem 1.1. Let $\boldsymbol{f}, \boldsymbol{f}_{t}, \boldsymbol{f}_{t t} \in C\left([0, T] ; \boldsymbol{L}^{2}(\Omega)\right), \boldsymbol{u}_{0} \in \boldsymbol{H}^{2}(\Omega) \cap \boldsymbol{H}_{0}^{1}(\Omega)$ and $\nabla \boldsymbol{u}_{0}=$ 0 . Then for any fixed $t_{0} \in(0, T)$, let $(\boldsymbol{u}, p)$ be the unique strong solution of (1.1)(1.2) in $\left[0, T_{1}\right]$ (with some $\left.T_{1} \leq T\right)$ and $\left(\boldsymbol{u}^{\varepsilon}, p^{\varepsilon}\right)$ be the solution of (1.8)-(1.9) with the initial data $\left(\boldsymbol{u}^{\varepsilon}\left(t_{0}\right), p^{\varepsilon}\left(t_{0}\right)\right)=\left(\boldsymbol{u}\left(t_{0}\right), p\left(t_{0}\right)\right)$. Then

$$
\begin{aligned}
\left\|\boldsymbol{u}-\boldsymbol{u}^{\varepsilon}\right\|_{L^{2}\left(t_{0}, T_{1} ; L^{2}\right)}^{2} & +\sqrt{\varepsilon}\left\|\boldsymbol{u}-\boldsymbol{u}^{\varepsilon}\right\|_{L^{\infty}\left(t_{0}, T_{1} ; \boldsymbol{L}^{2}\right)}^{2} \\
& +\varepsilon\left(\left\|\boldsymbol{u}-\boldsymbol{u}^{\varepsilon}\right\|_{L^{\infty}\left(t_{0}, T_{1} ; \boldsymbol{H}^{1}\right)}^{2}+\left\|p-p^{\varepsilon}\right\|_{L^{\infty}\left(t_{0}, T_{1} ; L^{2}\right)}^{2}\right) \leq C \varepsilon^{2},
\end{aligned}
$$

where $C$ is a constant depending on the data and $t_{0}$.

Since (1.6)-(1.7) is a second-order time discretization of (1.8)-(1.9) with $\varepsilon=\frac{1}{2} k^{2}$, in light of Theorem 1.1, we can speculate that the scheme (1.6)-(1.7), or any other similar discretization to (1.8)-(1.9), is of second order in $L^{2}\left(t_{0}, T_{1} ; \boldsymbol{L}^{2}\right)$ for the velocity and of first order in $L^{\infty}\left(t_{0}, T_{1} ; L^{2}\right)$ for the pressure. Therefore, instead of (1.6)-(1.7), we can also approximate (1.8)-(1.9) by the following scheme, which is of second order for the velocity and of first order for the pressure.

Given $\left(\boldsymbol{u}^{0}, p^{0}\right)$ in $\boldsymbol{H}_{0}^{1}(\Omega) \times H_{0}^{1}(\Omega) / \mathbb{R}$, we define $\left(\boldsymbol{u}^{n+1}, p^{n+1}\right)$ to be the solution of the system

$$
\begin{gathered}
\left\{\begin{array}{l}
\frac{\boldsymbol{u}^{n+1}-\boldsymbol{u}^{n}}{k}-\frac{\nu}{2} \Delta\left(\boldsymbol{u}^{n+1}+\boldsymbol{u}^{n}\right)+\tilde{B}\left(\frac{\boldsymbol{u}^{n+1}+\boldsymbol{u}^{n}}{2}, \frac{\boldsymbol{u}^{n+1}+\boldsymbol{u}^{n}}{2}\right)+\nabla p^{n}=\boldsymbol{f}\left(t_{n+\frac{1}{2}}\right), \\
\left.\boldsymbol{u}^{n+1}\right|_{\partial \Omega}=0
\end{array}\right. \\
\nabla \boldsymbol{u}^{n+1}-\beta k \Delta\left(p^{n+1}-p^{n}\right)=0,\left.\quad \frac{\partial p^{n+1}}{\partial \boldsymbol{n}}\right|_{\partial \Omega}=\left.\frac{\partial p^{n}}{\partial \boldsymbol{n}}\right|_{\partial \Omega}
\end{gathered}
$$

where $\beta$ is a constant to be determined. We will carry out a rigorous error analysis for the above scheme and indicate in $\S 4$ how the scheme (1.6)-(1.7) can be analyzed.

Although the projection step is never applied in (1.10)-(1.11), we will still refer to (1.10)-(1.11) as a projection scheme because of its similarity with (1.6)-(1.7). The key to the numerical efficiency and flexibility of the scheme (1.10)-(1.11) (resp. (1.6)-(1.7)) is the explicit treatment of the pressure in (1.10) (resp. (1.11)). As mentioned before, the nonlinear term in (1.10) (resp. (1.16)) is usually treated explicitly if the space variables are discretized; then at each time step, we only need to solve a vector Helmholtz equation and a scalar Poisson equation. In particular, fast Poisson solvers, if available, can be used. Furthermore, since the velocity and the pressure in a projection scheme are decoupled from each other, the space discretizations for the velocity and the pressure can be chosen independently, and they do not need to satisfy the Babuška-Brezzi inf-sup condition. In particular, one can use equal-order finite element or spectral element methods for the velocity and the pressure, which are otherwise incompatible in a conventional formulation.

In view of Theorem 1.1, we expect to prove the following error estimates for $(1.10)-(1.11)$ :

$$
k \sum_{n=1}^{m}\left\|\boldsymbol{u}\left(t_{n}\right)-\boldsymbol{u}^{n}\right\|^{2}+k^{2}\left\|\nabla\left(\boldsymbol{u}\left(t_{m}\right)-\boldsymbol{u}^{m}\right)\right\|^{2}+k^{2}\left\|p\left(t_{m}\right)-p^{m}\right\|^{2} \leq C k^{4}
$$

for all $1 \leq m \leq \frac{T_{1}-t_{0}}{k}$. It will be shown that the above estimates indeed hold provided suitable assumptions are made on the data for (1.1)-(1.2) and (1.10)(1.11) (cf. Theorem 3.1 for a precise statement of the results). 
In order to take advantage of the results in Theorem 1.1, it is natural to split the error $\boldsymbol{u}\left(t_{n}\right)-\boldsymbol{u}^{n}$ as $\left(\boldsymbol{u}\left(t_{n}\right)-\boldsymbol{u}^{\varepsilon}\left(t_{n}\right)\right)+\left(\boldsymbol{u}^{\varepsilon}\left(t_{n}\right)-\boldsymbol{u}^{n}\right)$ and try to derive a secondorder estimate for $\boldsymbol{u}^{\varepsilon}\left(t_{n}\right)-\boldsymbol{u}^{n}$. As usual, this process requires $\varepsilon$-independent a priori estimates for $\boldsymbol{u}_{t t t}^{\varepsilon}$ and $p_{t t}^{\varepsilon}$. Unfortunately, such estimates are not available (cf. Remark 3.2 in [14]). Therefore, we have to estimate $\boldsymbol{u}\left(t_{n}\right)-\boldsymbol{u}^{n}$ directly, as we did for $\boldsymbol{u}-\boldsymbol{u}^{\varepsilon}$ in [14]. However, this process becomes much more complex, owing to the additional difficulties introduced by the decoupled time discretization.

We note that the essential difficulty in the error estimation of projection schemes is associated with the approximation of the time-dependent linear Stokes operator. Although the treatment of the nonlinear term is very delicate and technical, the error introduced by the nonlinearity is relatively small compared to that introduced by the linear operator. In fact, the main results would remain the same should the nonlinear term be dropped from (1.1)-(1.2) and (1.10)-(1.11). Hence, the reader could skip the treatment of the nonlinear term at the first reading and thus obtain a clearer picture for the process of error analysis.

It appears that any decoupled time discretization for (1.8)-(1.9) can only be stable if $k^{2} \sim \varepsilon$ (cf. Remark 2.1). Hence, the accuracy of any decoupled time discretization scheme for (1.8)-(1.9) is dictated by the error estimate in Theorem 1.1 , which can only lead to second-order accuracy in $L^{2}\left(t_{0}, T_{1} ; \boldsymbol{L}^{2}\right)$ for the velocity, if no further restrictive conditions on the exact solution are assumed. This limited accuracy is due to the singular perturbation nature of (1.8)-(1.9), i.e., to the large error within the boundary layer introduced by the incompatible boundary condition $\left.\frac{\partial p_{t}^{\varepsilon}}{\partial \boldsymbol{n}}\right|_{\partial \Omega}=0$. Therefore, we can only expect a projection (or splitting) scheme to deliver higher than second-order accuracy if a more accurate boundary condition for the pressure is employed. We note that some interesting higher-order splitting schemes with improved pressure boundary conditions have been proposed in [11] and [9]. These schemes appear to achieve higher than second-order accuracy. Although some normal-mode analyses for a simple one-dimensional linear model were presented in $[\mathbf{1 1}]$ and $[\mathbf{9}]$, a rigorous analysis for more general cases is not yet available.

We now describe some of the notations used in this paper. We will use the standard notations $L^{2}(\Omega), H^{k}(\Omega)$ and $H_{0}^{k}(\Omega)$ to denote the usual Sobolev spaces over $\Omega$. The norm corresponding to $H^{k}(\Omega)$ will be denoted by $\|\cdot\|_{k}$. In particular, we will use $\|\cdot\|$ to denote the norm in $L^{2}(\Omega)$ and $(\cdot, \cdot)$ to denote the scalar product in $L^{2}(\Omega)$. The dual space of $H_{0}^{1}(\Omega)$ will be denoted by $H^{-1}(\Omega)$, and the duality between them will be denoted by $\langle\cdot, \cdot\rangle$. We will frequently use, without mention, the following norm equivalences:

$\|v\|_{1} \sim\|\nabla v\|, \forall v \in H_{0}^{1}(\Omega)$ or $H^{1}(\Omega) / \mathbb{R} ; \quad\|v\|_{2} \sim\|\Delta v\|, \forall v \in H^{2}(\Omega) \cap H_{0}^{1}(\Omega)$.

The vector functions and vector spaces will be denoted by boldface letters. To simplify the notation, we will omit the space variables from the notation, i.e., $\boldsymbol{v}(t)$ should be considered as a function of $t$ with value in a Sobolev space. We will use $C$ to denote a generic positive constant which may depend on the data and which may vary at different places.

The rest of the paper is organized as follows. In the next section, we prove the stability of the scheme and derive some additional a priori estimates for $\left(\boldsymbol{u}^{n}, p^{n}\right)$. In $\S 3$, we perform an error analysis for (1.10)-(1.11) by splitting the errors into 
two parts, one of which is associated with the linear operator and the other with the nonlinear term. In $\S 4$, we indicate how the results in $\S 3$ can be extended to some related projection schemes, and in $\S 5$ we present some numerical experiments which are in agreement with our analysis. An appendix is provided at the end for the estimation of the truncation errors and of the errors at the initial steps.

\section{Stability AND A PRIORI ESTimates}

We start by introducing some operators and relations for the treatment of the nonlinear terms. Denote

$$
\begin{gathered}
B(\boldsymbol{u}, \boldsymbol{v})=(\boldsymbol{u} \cdot \nabla) \boldsymbol{v}, \quad \tilde{B}(\boldsymbol{u}, \boldsymbol{v})=(\boldsymbol{u} \cdot \nabla) \boldsymbol{v}+\frac{1}{2}(\nabla \boldsymbol{u}) \boldsymbol{v}, \\
b(\boldsymbol{u}, \boldsymbol{v}, \boldsymbol{w})=(B(\boldsymbol{u}, \boldsymbol{v}), \boldsymbol{w}), \quad \tilde{b}(\boldsymbol{u}, \boldsymbol{v}, \boldsymbol{w})=(\tilde{B}(\boldsymbol{u}, \boldsymbol{v}), \boldsymbol{w}) .
\end{gathered}
$$

We note that

$$
b(\boldsymbol{u}, \boldsymbol{v}, \boldsymbol{v})=0, \quad \forall \boldsymbol{u} \in \boldsymbol{H}, \forall \boldsymbol{v} \in \boldsymbol{H}_{0}^{1}(\Omega)
$$

where $\boldsymbol{H}$ is defined in (1.5), and

$$
\tilde{b}(\boldsymbol{u}, \boldsymbol{v}, \boldsymbol{w})=\frac{1}{2}\{b(\boldsymbol{u}, \boldsymbol{v}, \boldsymbol{w})-b(\boldsymbol{u}, \boldsymbol{w}, \boldsymbol{v})\}, \forall \boldsymbol{u}, \boldsymbol{v}, \boldsymbol{w} \in \boldsymbol{H}_{0}^{1}(\Omega) .
$$

Therefore, we have

$$
\tilde{b}(\boldsymbol{u}, \boldsymbol{v}, \boldsymbol{v})=0, \quad \forall \boldsymbol{u}, \boldsymbol{v} \in \boldsymbol{H}_{0}^{1}(\Omega) .
$$

We will use occasionally the two inequalities below, which are valid for $d \leq 3$ and sharp for $d=3$ :

$$
\begin{gathered}
\tilde{b}(\boldsymbol{u}, \boldsymbol{v}, \boldsymbol{w}) \leq C\|\boldsymbol{u}\|_{1}\|\boldsymbol{v}\|_{1}^{\frac{1}{2}}\|\boldsymbol{v}\|_{2}^{\frac{1}{2}}\|\boldsymbol{w}\|, \forall \boldsymbol{v} \in \boldsymbol{H}^{2}(\Omega) \cap \boldsymbol{H}_{0}^{1}(\Omega), \boldsymbol{u}, \boldsymbol{w} \in \boldsymbol{H}_{0}^{1}(\Omega), \\
\tilde{b}(\boldsymbol{u}, \boldsymbol{v}, \boldsymbol{u}) \leq C\|\boldsymbol{u}\|^{\frac{1}{2}}\|\boldsymbol{u}\|_{1}^{\frac{3}{2}}\|\boldsymbol{v}\|_{1}, \forall \boldsymbol{u}, \boldsymbol{v} \in \boldsymbol{H}_{0}^{1}(\Omega) .
\end{gathered}
$$

In most cases, the following inequality, which is valid for $d \leq 4$, is sufficient for our purposes:

$$
\tilde{b}(\boldsymbol{u}, \boldsymbol{v}, \boldsymbol{w}) \leq\left\{\begin{array}{l}
\|\boldsymbol{u}\|_{1}\|\boldsymbol{v}\|_{1}\|\boldsymbol{w}\|_{1}, \forall \boldsymbol{u}, \boldsymbol{v}, \boldsymbol{w} \in \boldsymbol{H}_{0}^{1}(\Omega), \\
\|\boldsymbol{u}\|_{2}\|\boldsymbol{v}\|\|\boldsymbol{w}\|_{1}, \forall \boldsymbol{u} \in \boldsymbol{H}^{2}(\Omega) \cap \boldsymbol{H}_{0}^{1}(\Omega), \boldsymbol{v}, \boldsymbol{w} \in \boldsymbol{H}_{0}^{1}(\Omega), \\
\|\boldsymbol{u}\|_{2}\|\boldsymbol{v}\|_{1}\|\boldsymbol{w}\|, \forall \boldsymbol{u} \in \boldsymbol{H}^{2}(\Omega) \cap \boldsymbol{H}_{0}^{1}(\Omega), \boldsymbol{v}, \boldsymbol{w} \in \boldsymbol{H}_{0}^{1}(\Omega), \\
\|\boldsymbol{u}\|_{1}\|\boldsymbol{v}\|_{2}\|\boldsymbol{w}\|, \forall \boldsymbol{v} \in \boldsymbol{H}^{2}(\Omega) \cap \boldsymbol{H}_{0}^{1}(\Omega), \boldsymbol{u}, \boldsymbol{w} \in \boldsymbol{H}_{0}^{1}(\Omega) .
\end{array}\right.
$$

These inequalities can be proved by using (2.2), Hölder's inequality and Sobolev inequalities (see, for instance, Lemma 2.1 in [21]).

The following lemma of Gronwall type will be repeatedly used (see, for instance, [8] for a proof). 
Lemma 2.1 (Discrete Gronwall lemma). Let $y^{n}, h^{n}, g^{n}, f^{n}$ be nonnegative sequences satisfying

$$
y^{m}+k \sum_{n=0}^{m} h^{n} \leq B+k \sum_{n=0}^{m}\left(g^{n} y^{n}+f^{n}\right), \text { with } k \sum_{n=0}^{\left[\frac{T}{k}\right]} g^{n} \leq M, \forall 0 \leq m \leq \frac{T}{k} .
$$

Assume $k g^{n}<1$ and let $\sigma=\max _{0 \leq n \leq \frac{T}{k}}\left(1-k g^{n}\right)^{-1}$. Then

$$
y^{m}+k \sum_{n=1}^{m} h^{n} \leq \exp (\sigma M)\left(B+k \sum_{n=0}^{m} f^{n}\right), \quad \forall m \leq \frac{T}{k} .
$$

2.1. Stability. We first establish a stability result for the scheme (1.10)-(1.11). The techniques used here will be repeatedly used later in different circumstances.

Lemma 2.2. Let $\beta \geq \frac{1}{4}$. Then there exists $C>0$ such that for all $m$ with $1 \leq m \leq \frac{T}{k}-1$

$$
\begin{aligned}
\left(1-\frac{1}{4 \beta}\right)\left\|\boldsymbol{u}^{m+1}\right\|^{2} & +k \sum_{n=1}^{m}\left\|\nabla\left(\boldsymbol{u}^{n+1}+\boldsymbol{u}^{n}\right)\right\|^{2} \\
& \leq C\left(\left\|\boldsymbol{u}^{1}\right\|^{2}+k^{2}\left\|\nabla p^{0}\right\|^{2}+k^{2}\left\|\nabla p^{1}\right\|^{2}+\|\boldsymbol{f}\|_{C\left([0, T] ; \boldsymbol{H}^{-1}\right)}^{2}\right) .
\end{aligned}
$$

Proof. We derive from (1.11) that

$$
\nabla\left(\boldsymbol{u}^{n+1}+\boldsymbol{u}^{n}\right)-\beta k \Delta\left(p^{n+1}-p^{n-1}\right)=0,\left.\quad \frac{\partial p^{n+1}}{\partial \boldsymbol{n}}\right|_{\partial \Omega}=\left.\frac{\partial p^{n-1}}{\partial \boldsymbol{n}}\right|_{\partial \Omega} .
$$

Taking the inner product of (1.10) with $k\left(\boldsymbol{u}^{n+1}+\boldsymbol{u}^{n}\right)$ and of (2.7) with $k p^{n}$ and summing up the two relations, thanks to (2.3), we derive

$$
\begin{aligned}
\left\|\boldsymbol{u}^{n+1}\right\|^{2} & -\left\|\boldsymbol{u}^{n}\right\|^{2}+\frac{\nu k}{2}\left\|\nabla\left(\boldsymbol{u}^{n+1}+\boldsymbol{u}^{n}\right)\right\|^{2}+\beta k^{2}\left(\nabla\left(p^{n+1}-p^{n-1}\right), \nabla p^{n}\right) \\
& =k\left\langle\boldsymbol{u}^{n+1}+\boldsymbol{u}^{n}, \boldsymbol{f}\left(t_{n+\frac{1}{2}}\right)\right\rangle \\
& \leq \frac{\nu k}{4}\left\|\nabla\left(\boldsymbol{u}^{n+1}+\boldsymbol{u}^{n}\right)\right\|^{2}+C k\left\|\boldsymbol{f}\left(t_{n+\frac{1}{2}}\right)\right\|_{-1}^{2} .
\end{aligned}
$$

Using the algebraic relations below,

$$
\begin{aligned}
& (a-b, a)=\frac{1}{2}\left(|a|^{2}-|b|^{2}+|b-a|^{2}\right), \\
& (a-b, b)=\frac{1}{2}\left(|a|^{2}-|b|^{2}-|b-a|^{2}\right),
\end{aligned}
$$

we find that

$$
\begin{aligned}
\left(\nabla\left(p^{n+1}-p^{n-1}\right), \nabla p^{n}\right)= & \frac{1}{2}\left\{\left\|\nabla p^{n+1}\right\|^{2}-\left\|\nabla p^{n-1}\right\|^{2}\right\} \\
& +\frac{1}{2}\left\{\left\|\nabla\left(p^{n}-p^{n-1}\right)\right\|^{2}-\left\|\nabla\left(p^{n+1}-p^{n}\right)\right\|^{2}\right\} .
\end{aligned}
$$


By summing up (2.1) for $n=1$ to $m$, we derive that

$$
\begin{aligned}
\left\|\boldsymbol{u}^{m+1}\right\|^{2}+\frac{\nu k}{4} \sum_{n=1}^{m}\left\|\nabla\left(\boldsymbol{u}^{n+1}+\boldsymbol{u}^{n}\right)\right\|^{2}+\frac{\beta k^{2}}{2}\left(\left\|\nabla p^{m+1}\right\|^{2}+\left\|\nabla p^{m}\right\|^{2}\right) \\
\leq\left\|\boldsymbol{u}^{1}\right\|^{2}+\|\boldsymbol{f}\|_{C\left([0, T] ; \boldsymbol{H}^{-1}\right)}^{2}+\frac{\beta k^{2}}{2}\left(\left\|\nabla p^{1}\right\|^{2}+\left\|\nabla p^{0}\right\|^{2}\right) \\
+\frac{\beta k^{2}}{2}\left\|\nabla\left(p^{m+1}-p^{m}\right)\right\|^{2} .
\end{aligned}
$$

From (1.11),

$$
\beta k^{2}\left\|\nabla\left(p^{m+1}-p^{m}\right)\right\|^{2} \leq \frac{1}{\beta}\left\|\boldsymbol{u}^{m+1}\right\|^{2} .
$$

Therefore,

$$
\begin{aligned}
\frac{\beta k^{2}}{2}\left\|\nabla\left(p^{m+1}-p^{m}\right)\right\|^{2} & \leq \frac{1}{4 \beta}\left\|\boldsymbol{u}^{m+1}\right\|^{2}+\frac{1}{4} \beta k^{2}\left\|\nabla\left(p^{m+1}-p^{m}\right)\right\|^{2} \\
& \leq \frac{1}{4 \beta}\left\|\boldsymbol{u}^{m+1}\right\|^{2}+\frac{1}{2} \beta k^{2}\left(\left\|\nabla p^{m+1}\right\|^{2}+\left\|\nabla p^{m}\right\|^{2}\right) .
\end{aligned}
$$

The above inequality and (2.11) imply that

$$
\begin{aligned}
\left(1-\frac{1}{4 \beta}\right)\left\|\boldsymbol{u}^{m+1}\right\|^{2} & +\frac{\nu k}{4} \sum_{n=1}^{m}\left\|\nabla\left(\boldsymbol{u}^{n+1}+\boldsymbol{u}^{n}\right)\right\|^{2} \\
& \leq 2\left\|\boldsymbol{u}^{1}\right\|^{2}+\frac{\beta k^{2}}{2}\left(\left\|\nabla p^{1}\right\|^{2}+\left\|\nabla p^{0}\right\|^{2}\right)+\|\boldsymbol{f}\|_{C\left([0, T] ; \boldsymbol{H}^{-1}\right)}^{2} .
\end{aligned}
$$

Remark 2.1. From the above proof and our numerical experiences, it appears that the scheme becomes unstable if $\beta<\frac{1}{4}$. This, in particular, prevents us from increasing the accuracy by setting $\beta=k^{\alpha}$ for some $\alpha>0$ in (1.10)-(1.11). On the other hand, since the truncation error increases as $\beta$ increases, it is advised to use $\beta=\frac{1}{4}$.

2.2. Some additional a priori estimates. We note that Lemma 2.2 does not provide a $k$-independent stability result for $\left\|\nabla p^{n}\right\|$ and is not sufficient for obtaining any meaningful error estimate. Further $k$-independent stability results, especially for $\left\|\nabla p^{n}\right\|$, are required to conduct an error analysis. We can derive desired $k$ independent stability results by choosing the initial data $\left(\boldsymbol{u}^{0}, p^{0}\right)$ for $(1.10)-(1.11)$ to be an approximation of the solution $\left(\boldsymbol{u}\left(t_{0}\right), p\left(t_{0}\right)\right)$ for some $t_{0}>0$.

In the rest of the paper, we fix $t_{0}>0$ and assume that we are given an initial data $\left(\boldsymbol{u}^{0}, p^{0}\right)$ such that

$$
\left\|\boldsymbol{u}^{0}-\boldsymbol{u}\left(t_{0}\right)\right\| \leq C k^{2}, \quad\left\|\nabla\left(\boldsymbol{u}^{0}-\boldsymbol{u}\left(t_{0}\right)\right)\right\|+\left\|\nabla\left(p^{0}-p\left(t_{0}\right)\right)\right\| \leq C k .
$$

Remark 2.2. It is well known that the solution $(\boldsymbol{u}(t), p(t))$ of the Navier-Stokes equations (1.1)-(1.2) is smooth at $t=0$ only if the data $\boldsymbol{u}(0)$ and $\boldsymbol{f}(0)$ satisfy certain nonlocal compatibility conditions (cf. [7]). To avoid assuming these nonlocal compatibility conditions, we opt to start the scheme (1.10)-(1.11) at time $t_{0}>0$ 
with an initial data $\left(\boldsymbol{u}^{0}, p^{0}\right)$ satisfying (2.12). Such an initial condition can be obtained, for instance, by using a standard coupled scheme.

We should point out that this initialization process may not always be necessary since many schemes have the ability to damp out the large initial errors, owing to the singularity at the initial time (cf. [3]).

As usual in an error analysis, we need some regularity results for solutions of the Navier-Stokes equations (1.1)-(1.2). Let $V=\left\{\boldsymbol{v} \in \boldsymbol{H}_{0}^{1}(\Omega): \nabla \boldsymbol{v}=0\right\}$; it is well known that (see, for instance, $[\mathbf{7}]$ ) for

$$
\boldsymbol{u}_{0} \in \boldsymbol{H}^{2}(\Omega) \cap \boldsymbol{V}, \quad \boldsymbol{f} \in C\left([0, T] ; \boldsymbol{L}^{2}(\Omega)\right),
$$

there exists $T_{1} \leq T$ ( $T_{1}=T$ if $\left.d=2\right)$ such that the solution of (1.1)-(1.2) satisfies

$$
\|\boldsymbol{u}(t)\|_{2}+\left\|\boldsymbol{u}_{t}(t)\right\|+\|p(t)\|_{1} \leq C, \forall t \in\left[0, T_{1}\right] .
$$

Although higher regularity at $t=0$ requires that the data $\boldsymbol{u}_{0}$ and $\boldsymbol{f}(0)$ satisfy certain nonlocal compatibility conditions, the solution becomes as smooth as the data allows for $t>0$ thanks to the smoothing property of the Navier-Stokes equations. In particular, we have the following regularity result, which is sufficient for our error analysis (see, for instance, Theorem 2.4 in [7]).

Proposition 2.1. In addition to (2.13), we assume that

$$
\boldsymbol{f}_{t}, \boldsymbol{f}_{t t} \in C\left([0, T] ; \boldsymbol{L}^{2}(\Omega)\right) .
$$

Then for any $t_{0} \in\left(0, T_{1}\right)$, the solution of (1.1)-(1.2) satisfies

$$
\begin{aligned}
\left\|\boldsymbol{u}_{t t}\right\|^{2} & +\left\|\boldsymbol{u}_{t}(t)\right\|_{2}^{2}+\left\|p_{t}(t)\right\|_{1}^{2} \\
& +\int_{t_{0}}^{t}\left(\left\|\boldsymbol{u}_{t t t}(s)\right\|^{2}+\left\|\boldsymbol{u}_{t t}(s)\right\|_{2}^{2}+\left\|p_{t t}(s)\right\|_{1}^{2}\right) d s \leq C, \forall t \in\left[t_{0}, T_{1}\right] .
\end{aligned}
$$

To simplify the notation, we denote hereafter $t_{\alpha}=t_{0}+\alpha k$ and

$$
\tilde{w}\left(t_{n+\frac{1}{2}}\right)=\frac{1}{2}\left(w\left(t_{n+1}\right)+w\left(t_{n}\right)\right), \quad \tilde{a}^{n+\frac{1}{2}}=\frac{1}{2}\left(a^{n+1}+a^{n}\right)
$$

for any function $w(t)$ and any sequence $\left\{a^{n}\right\}$. We denote also $M=\left[\frac{T_{1}-t_{0}}{k}\right]$, the integer part of $\frac{T_{1}-t_{0}}{k}$.

We shall derive some a priori estimates on $\left(\boldsymbol{u}^{n}, p^{n}\right)$ and some crude error estimates which will be used later.

Lemma 2.3. Assume (2.13) and (2.15). Then, given $\beta>\frac{1}{4}$ and the initial data satisfying (2.12), we have

$$
\left\|\nabla \boldsymbol{u}^{m+1}\right\|^{2}+\left\|\Delta\left(\boldsymbol{u}^{m+1}+\boldsymbol{u}^{m}\right)\right\|^{2}+\left\|\nabla p^{m+1}\right\|^{2} \leq C, \forall 0 \leq m \leq M-1 .
$$

Proof. We denote $\boldsymbol{e}^{n}=\boldsymbol{u}\left(t_{n}\right)-\boldsymbol{u}^{n}$ and $q^{n}=p\left(t_{n}\right)-p^{n}$. Subtracting (1.10)-(1.11) from (1.1)-(1.2), we obtain the error equations

$$
\frac{\boldsymbol{e}^{n+1}-\boldsymbol{e}^{n}}{k}-\frac{\nu}{2} \Delta\left(\boldsymbol{e}^{n+1}+\boldsymbol{e}^{n}\right)+\nabla q^{n}=\boldsymbol{R}^{n}+\boldsymbol{Q}^{n}
$$




$$
\begin{aligned}
\left(\nabla \boldsymbol{e}^{n+1}, \gamma\right) & +\beta k\left(\nabla\left(q^{n+1}-q^{n}\right), \nabla \gamma\right) \\
& =\beta k\left(\nabla\left(p\left(t_{n+1}\right)-p\left(t_{n}\right)\right), \nabla \gamma\right), \forall \gamma \in H^{1}(\Omega) / \mathbb{R}
\end{aligned}
$$

or

$$
\begin{aligned}
\left(\nabla\left(\boldsymbol{e}^{n+1}+\boldsymbol{e}^{n}\right), \gamma\right) & +\beta k\left(\nabla\left(q^{n+1}-q^{n-1}\right), \nabla \gamma\right) \\
& =\beta k\left(\nabla\left(p\left(t_{n+1}\right)-p\left(t_{n-1}\right)\right), \nabla \gamma\right), \forall \gamma \in H^{1}(\Omega) / \mathbb{R}
\end{aligned}
$$

where

$$
\begin{aligned}
\boldsymbol{Q}^{n} & =\tilde{B}\left(\tilde{\boldsymbol{u}}^{n+\frac{1}{2}}, \tilde{\boldsymbol{u}}^{n+\frac{1}{2}}\right)-\tilde{B}\left(\tilde{\boldsymbol{u}}\left(t_{n+\frac{1}{2}}\right), \tilde{\boldsymbol{u}}\left(t_{n+\frac{1}{2}}\right)\right) \\
& =-\tilde{B}\left(\tilde{\boldsymbol{u}}^{n+\frac{1}{2}}, \tilde{\boldsymbol{e}}^{n+\frac{1}{2}}\right)-\tilde{B}\left(\tilde{\boldsymbol{e}}^{n+\frac{1}{2}}, \tilde{\boldsymbol{u}}\left(t_{n+\frac{1}{2}}\right)\right)
\end{aligned}
$$

is the error related to the nonlinear terms and $\boldsymbol{R}^{n}$ is the truncation error defined by

$$
\begin{aligned}
\boldsymbol{R}^{n}= & \frac{\boldsymbol{u}\left(t_{n+1}\right)-\boldsymbol{u}\left(t_{n}\right)}{k}-\frac{\nu}{2} \Delta\left(\boldsymbol{u}\left(t_{n+1}\right)+\boldsymbol{u}\left(t_{n}\right)\right) \\
& \quad+B\left(\tilde{\boldsymbol{u}}\left(t_{n+\frac{1}{2}}\right), \tilde{\boldsymbol{u}}\left(t_{n+\frac{1}{2}}\right)\right)+\nabla p\left(t_{n}\right) \\
= & \frac{\boldsymbol{u}\left(t_{n+1}\right)-\boldsymbol{u}\left(t_{n}\right)}{k}-\frac{\nu}{2} \Delta\left(\boldsymbol{u}\left(t_{n+1}\right)+\boldsymbol{u}\left(t_{n}\right)\right)+B\left(\tilde{\boldsymbol{u}}\left(t_{n+\frac{1}{2}}\right), \tilde{\boldsymbol{u}}\left(t_{n+\frac{1}{2}}\right)\right) \\
& \quad+\nabla p\left(t_{n+\frac{1}{2}}\right)+\left(\nabla p\left(t_{n}\right)-\nabla p\left(t_{n+\frac{1}{2}}\right)\right) \equiv \boldsymbol{R}_{1}^{n}+\boldsymbol{R}_{2}^{n},
\end{aligned}
$$

where $\boldsymbol{R}_{1}^{n}$ (resp. $\boldsymbol{R}_{2}^{n}$ ) corresponds to the second-order (resp. first-order) part of $\boldsymbol{R}^{n}$.

Taking the inner product of (2.17) with $k\left(\boldsymbol{e}^{n+1}+\boldsymbol{e}^{n}\right)=2 k \tilde{\boldsymbol{e}}^{n+\frac{1}{2}}$ and of (2.19) with $k q^{n}$, and summing up the two relations, we obtain

$$
\begin{aligned}
\left\|\boldsymbol{e}^{n+1}\right\|^{2}- & \left\|\boldsymbol{e}^{n}\right\|^{2}+\frac{\nu k}{2}\left\|\nabla\left(\boldsymbol{e}^{n+1}+\boldsymbol{e}^{n}\right)\right\|^{2}+\beta k^{2}\left(\nabla\left(q^{n+1}-q^{n-1}\right), \nabla q^{n}\right) \\
= & k\left\langle\boldsymbol{e}^{n+1}+\boldsymbol{e}^{n}, \boldsymbol{R}^{n}\right\rangle-2 k \tilde{b}\left(\tilde{\boldsymbol{e}}^{n+\frac{1}{2}}, \tilde{\boldsymbol{u}}\left(t_{n+\frac{1}{2}}\right), \tilde{\boldsymbol{e}}^{n+\frac{1}{2}}\right) \\
& +\beta k^{2}\left(\nabla\left(p\left(t_{n+1}\right)-p\left(t_{n-1}\right)\right), \nabla q^{n}\right) .
\end{aligned}
$$

We infer from (2.9) that

$$
\begin{aligned}
\beta k^{2}\left(\nabla\left(q^{n+1}-q^{n-1}\right),\right. & \left.\nabla q^{n}\right)=\frac{\beta k^{2}}{2}\left\{\left\|\nabla q^{n+1}\right\|^{2}-\left\|\nabla q^{n-1}\right\|^{2}\right\} \\
+ & \frac{\beta k^{2}}{2}\left\{\left\|\nabla\left(q^{n}-q^{n-1}\right)\right\|^{2}-\left\|\nabla\left(q^{n+1}-q^{n}\right)\right\|^{2}\right\} .
\end{aligned}
$$

The terms on the right-hand side of $(2.22)$ can be handled as follows:

$$
\begin{gathered}
k\left\langle\boldsymbol{R}^{n}, \boldsymbol{e}^{n+1}+\boldsymbol{e}^{n}\right\rangle \leq \frac{\nu k}{8}\left\|\nabla\left(\boldsymbol{e}^{n+1}+\boldsymbol{e}^{n}\right)\right\|^{2}+C k\left\|\boldsymbol{R}^{n}\right\|_{-1}^{2}, \\
\beta k^{2}\left(\nabla\left(p\left(t_{n+1}\right)-p\left(t_{n-1}\right)\right), \nabla q^{n}\right) \leq k^{3}\left\|\nabla q^{n}\right\|^{2}+C k\left\|\nabla\left(p\left(t_{n+1}\right)-p\left(t_{n-1}\right)\right)\right\|^{2} .
\end{gathered}
$$


Using (2.6) and (2.14), we get

$$
\begin{aligned}
4 k \tilde{b}\left(\tilde{\boldsymbol{e}}^{n+\frac{1}{2}}, \tilde{\boldsymbol{u}}\left(t_{n+\frac{1}{2}}\right), \tilde{\boldsymbol{e}}^{n+\frac{1}{2}}\right) & \leq C k\left\|\tilde{\boldsymbol{u}}\left(t_{n+\frac{1}{2}}\right)\right\|_{2}\left\|\tilde{\boldsymbol{e}}^{n+\frac{1}{2}}\right\|\left\|\nabla \tilde{\boldsymbol{e}}^{n+\frac{1}{2}}\right\| \\
& \leq \frac{\nu k}{8}\left\|\nabla\left(\boldsymbol{e}^{n+1}+\boldsymbol{e}^{n}\right)\right\|^{2}+C k\left\|\tilde{\boldsymbol{e}}^{n+\frac{1}{2}}\right\|^{2} .
\end{aligned}
$$

Taking the sum of (2.22) for $n=1$ to $m$, and using the above relations, we arrive at

$$
\begin{aligned}
\left\|\boldsymbol{e}^{m+1}\right\|^{2} & +\frac{\nu k}{4} \sum_{n=1}^{m}\left\|\nabla\left(\boldsymbol{e}^{n+1}+\boldsymbol{e}^{n}\right)\right\|^{2}+\frac{\beta k^{2}}{2}\left(\left\|\nabla q^{m+1}\right\|^{2}+\left\|\nabla q^{m}\right\|^{2}\right) \\
\leq & \left\|\boldsymbol{e}^{1}\right\|^{2}+\frac{\beta k^{2}}{2}\left(\left\|\nabla q^{1}\right\|^{2}+\left\|\nabla q^{0}\right\|^{2}+\left\|\nabla\left(q^{m+1}-q^{m}\right)\right\|^{2}\right) \\
& +C k \sum_{n=1}^{m}\left(\left\|\boldsymbol{R}^{n}\right\|_{-1}^{2}+\left\|\tilde{\boldsymbol{e}}^{n+\frac{1}{2}}\right\|^{2}+k^{2}\left\|\nabla q^{n}\right\|^{2}\right)+C k^{2}\left\|p_{t}\right\|_{C\left([0, T] ; H^{1}(\Omega)\right)}^{2}
\end{aligned}
$$

(thanks to (2.14), (2.12) and Lemmas A1 and A2 in the Appendix)

$$
\leq C k^{2}+C k \sum_{n=1}^{m}\left(\left\|\boldsymbol{e}^{n+1}\right\|^{2}+k^{2}\left\|\nabla q^{n}\right\|^{2}\right)+\frac{\beta k^{2}}{2}\left\|\nabla\left(q^{m+1}-q^{m}\right)\right\|^{2} .
$$

The last term on the right-hand side needs to be treated with special care. Let $\delta=\beta-\frac{1}{4}>0$ and $p\left(t_{m+1}\right)-p\left(t_{m}\right)=k p_{t}\left(\xi_{m}\right)$. Taking the inner product of (2.18) with $q^{n+1}-q^{n}$, we obtain

$$
\begin{aligned}
\beta k \nabla\left(q^{m+1}-q^{m}\right) \|^{2}= & \left(\boldsymbol{e}^{m+1}, \nabla\left(q^{m+1}-q^{m}\right)\right) \\
& +\beta k\left(\nabla\left(p\left(t_{m+1}\right)-p\left(t_{m}\right)\right), \nabla\left(q^{m+1}-q^{m}\right)\right) \\
\leq & \left(\frac{\beta}{2}-\frac{3 \delta}{8}\right) k\left\|\nabla\left(q^{m+1}-q^{m}\right)\right\|^{2}+\frac{1}{4 k\left(\frac{\beta}{2}-\frac{3 \delta}{8}\right)}\left\|\boldsymbol{e}^{m+1}\right\|^{2} \\
& +\frac{3 \delta k}{8}\left\|\nabla\left(q^{m+1}-q^{m}\right)\right\|^{2}+C k\left\|\nabla\left(p\left(t_{m+1}\right)-p\left(t_{m}\right)\right)\right\|^{2} \\
= & \frac{\beta k}{2}\left\|\nabla\left(q^{m+1}-q^{m}\right)\right\|^{2}+\frac{2}{(\delta+1) k}\left\|\boldsymbol{e}^{m+1}\right\|^{2}+C k^{3}\left\|\nabla p_{t}\left(\xi_{m}\right)\right\|^{2} .
\end{aligned}
$$

We then derive from the above inequality and (2.16)

$$
\beta k^{2}\left\|\nabla\left(q^{m+1}-q^{m}\right)\right\|^{2} \leq \frac{4}{\delta+1}\left\|\boldsymbol{e}^{m+1}\right\|^{2}+C k^{4} .
$$

Hence,

$$
\begin{aligned}
\frac{\beta k^{2}}{2}\left\|\nabla\left(q^{m+1}-q^{m}\right)\right\|^{2}= & \frac{\left(1+\frac{\delta}{2}\right) \beta k^{2}}{4}\left\|\nabla\left(q^{m+1}-q^{m}\right)\right\|^{2} \\
& +\frac{\left(1-\frac{\delta}{2}\right) \beta k^{2}}{4}\left\|\nabla\left(q^{m+1}-q^{m}\right)\right\|^{2} \\
\leq & \frac{1+\frac{\delta}{2}}{1+\delta}\left\|e^{m+1}\right\|^{2}+C k^{4} \\
& +\frac{\left(1-\frac{\delta}{2}\right) \beta k^{2}}{2}\left(\left\|\nabla q^{m+1}\right\|^{2}+\left\|\nabla q^{m}\right\|^{2}\right) .
\end{aligned}
$$


We derive from the above inequality and (2.24) that

$$
\begin{aligned}
\frac{\delta}{2(1+\delta)}\left\|\boldsymbol{e}^{m+1}\right\|^{2}+\frac{\nu k}{4} \sum_{n=1}^{m} \| \nabla\left(\boldsymbol{e}^{n+1}+\right. & \left.\boldsymbol{e}^{n}\right) \|^{2}+\frac{\delta \beta k^{2}}{4}\left(\left\|\nabla q^{m+1}\right\|^{2}+\left\|\nabla q^{m}\right\|^{2}\right) \\
& \leq C k^{2}+C k \sum_{n=1}^{m}\left(\left\|\boldsymbol{e}^{n+1}\right\|^{2}+k^{2}\left\|\nabla q^{n}\right\|^{2}\right) .
\end{aligned}
$$

Applying Lemma 2.1 with $y^{n}=\left\|\boldsymbol{e}^{n+1}\right\|^{2}+k^{2}\left\|\nabla q^{n}\right\|^{2}$ to the above inequality, we obtain

$$
\left\|\boldsymbol{e}^{m+1}\right\|^{2}+k \sum_{n=1}^{m}\left\|\nabla\left(\boldsymbol{e}^{n+1}+\boldsymbol{e}^{n}\right)\right\|^{2}+k^{2}\left\|\nabla q^{m+1}\right\|^{2} \leq C k^{2}, \forall 1 \leq m \leq M-1
$$

In view of (2.14), the above inequality implies in particular that

$$
\left\|\nabla\left(\boldsymbol{u}^{n+1}+\boldsymbol{u}^{n}\right)\right\|^{2}+\left\|\nabla p^{n}\right\|^{2} \leq C, \forall 1 \leq n \leq M-1 .
$$

We now consider the term $\nabla p^{n}$ in (1.10) as a source term and take the scalar product of (1.10) with $-2 k \Delta\left(\boldsymbol{u}^{n+1}+\boldsymbol{u}^{n}\right)$; denoting $\boldsymbol{g}^{n}=\boldsymbol{f}\left(t_{n+\frac{1}{2}}\right)-\nabla p^{n}$ and using (2.4), we obtain

$$
\begin{aligned}
2\left\|\nabla \boldsymbol{u}^{n+1}\right\|^{2}-2\left\|\nabla \boldsymbol{u}^{n}\right\|^{2} & +k \nu\left\|\Delta\left(\boldsymbol{u}^{n+1}+\boldsymbol{u}^{n}\right)\right\|^{2} \\
& =2 k\left(\boldsymbol{g}^{n}, \Delta\left(\boldsymbol{u}^{n+1}+\boldsymbol{u}^{n}\right)\right)+4 k \tilde{b}\left(\tilde{\boldsymbol{u}}^{n+\frac{1}{2}}, \tilde{\boldsymbol{u}}^{n+\frac{1}{2}}, \Delta \tilde{\boldsymbol{u}}^{n+\frac{1}{2}}\right) \\
& \leq 2 k\left(\boldsymbol{g}^{n}, \Delta\left(\boldsymbol{u}^{n+1}+\boldsymbol{u}^{n}\right)\right)+C k\left\|\tilde{\boldsymbol{u}}^{n+\frac{1}{2}}\right\|_{1}^{\frac{3}{2}}\left\|\Delta \tilde{\boldsymbol{u}}^{n+\frac{1}{2}}\right\|^{\frac{3}{2}} \\
& \leq \frac{\nu k}{2}\left\|\Delta\left(\boldsymbol{u}^{n+1}+\boldsymbol{u}^{n}\right)\right\|^{2}+C k\left\|\boldsymbol{g}^{n}\right\|^{2}+C k\left\|\tilde{\boldsymbol{u}}^{n+\frac{1}{2}}\right\|_{1}^{6} .
\end{aligned}
$$

Summing up the above relation over $n$, using (2.27), we derive that

$$
\left\|\nabla \boldsymbol{u}^{m+1}\right\|^{2}+k \nu \sum_{n=1}^{m}\left\|\Delta\left(\boldsymbol{u}^{n+1}+\boldsymbol{u}^{n}\right)\right\|^{2} \leq C, \forall 1 \leq m \leq M-1 .
$$

Taking the inner product of $(2.17)$ with $-\Delta \tilde{\boldsymbol{e}}^{n+\frac{1}{2}}$, thanks to (2.26) and Lemma A1, we get

$$
\left\|\Delta \tilde{\boldsymbol{e}}^{n+\frac{1}{2}}\right\|^{2} \leq C+C\left(\boldsymbol{Q}^{n},-\Delta \tilde{\boldsymbol{e}}^{n+\frac{1}{2}}\right) .
$$

On the other hand, using (2.4) and (2.6), we derive from (2.20) that

$$
\begin{aligned}
\left(\boldsymbol{Q}^{n}, \tilde{\boldsymbol{e}}^{n+\frac{1}{2}}\right)= & -\tilde{b}\left(\tilde{\boldsymbol{u}}^{n+\frac{1}{2}}, \tilde{\boldsymbol{e}}^{n+\frac{1}{2}}, \Delta \tilde{\boldsymbol{e}}^{n+\frac{1}{2}}\right)-\tilde{B}\left(\tilde{\boldsymbol{e}}^{n+\frac{1}{2}}, \tilde{\boldsymbol{u}}\left(t_{n+\frac{1}{2}}\right), \Delta \tilde{\boldsymbol{e}}^{n+\frac{1}{2}}\right) \\
\leq & C\left\|\tilde{\boldsymbol{u}}^{n+\frac{1}{2}}\right\|_{1}\left\|\tilde{\boldsymbol{e}}^{n+\frac{1}{2}}\right\|_{1}^{\frac{1}{2}}\left\|\Delta \tilde{\boldsymbol{e}}^{n+\frac{1}{2}}\right\|^{\frac{3}{2}}+\left\|\tilde{\boldsymbol{e}}^{n+\frac{1}{2}}\right\|_{1}\left\|\tilde{\boldsymbol{u}}\left(t_{n+\frac{1}{2}}\right)\right\|_{2}\left\|\Delta \tilde{\boldsymbol{e}}^{n+\frac{1}{2}}\right\| \\
& (\text { by using Young's inequality, }(2.14) \text { and }(2.28)) \\
\leq & \frac{1}{2}\left\|\Delta \tilde{\boldsymbol{e}}^{n+\frac{1}{2}}\right\|^{2}+C, \forall 1 \leq n \leq M-1 .
\end{aligned}
$$

Therefore, $\left\|\Delta \tilde{\boldsymbol{e}}^{n+\frac{1}{2}}\right\| \leq C, \forall 1 \leq n \leq M-1$. 
Lemma 2.4. Under the assumption of Lemma 2.3, we have

$$
\left\|\boldsymbol{e}^{n+1}-\boldsymbol{e}^{n}\right\|+k\left\|\nabla\left(p^{n+1}-p^{n}\right)\right\| \leq C k^{2}, \forall 1 \leq n \leq M-1 .
$$

Proof. Denote

$$
\begin{aligned}
& \varepsilon^{n}=e^{n}-e^{n-1}, \quad \boldsymbol{w}^{n}=\boldsymbol{u}^{n}-\boldsymbol{u}^{n-1}, \quad r^{n}=q^{n}-q^{n-1}, \\
& \boldsymbol{E}_{r}^{n}=\boldsymbol{R}^{n}-\boldsymbol{R}^{n-1}, \quad \boldsymbol{E}_{q}^{n}=\boldsymbol{Q}^{n}-\boldsymbol{Q}^{n-1}, \\
& E_{p}^{n}=\left(p\left(t_{n+1}\right)-p\left(t_{n}\right)\right)-\left(p\left(t_{n-1}\right)-p\left(t_{n-2}\right)\right) .
\end{aligned}
$$

We derive from (2.17)-(2.19) that for $n \geq 2$

$$
\begin{aligned}
& \frac{\varepsilon^{n+1}-\varepsilon^{n}}{k}-\frac{\nu}{2} \Delta\left(\varepsilon^{n+1}+\varepsilon^{n}\right)+\nabla r^{n}=\boldsymbol{E}_{r}^{n}+\boldsymbol{E}_{q}^{n}, \\
& \begin{aligned}
\left(\nabla\left(\varepsilon^{n+1}+\varepsilon^{n}\right), \gamma\right) & +\beta k\left(\nabla\left(r^{n+1}-r^{n-1}\right), \nabla \gamma\right) \\
& =\beta k\left(\nabla E_{p}^{n}, \nabla \gamma\right), \forall \gamma \in H^{1}(\Omega) / \mathbb{R} .
\end{aligned}
\end{aligned}
$$

Taking the inner product of (2.30) with $k\left(\varepsilon^{n+1}+\varepsilon^{n}\right)=2 k \tilde{\varepsilon}^{n+\frac{1}{2}}$ and setting $\gamma=k r^{n}$ in (2.31), and summing up the two relations, we obtain

$$
\begin{aligned}
\left\|\varepsilon^{n+1}\right\|^{2} & -\left\|\varepsilon^{n}\right\|^{2}+2 \nu k\left\|\nabla \tilde{\varepsilon}^{n+\frac{1}{2}}\right\|^{2}+\beta k^{2}\left(\nabla\left(r^{n+1}-r^{n-1}\right), \nabla r^{n}\right) \\
& =2 k\left(\tilde{\varepsilon}^{n+\frac{1}{2}}, \boldsymbol{E}_{r}^{n}+\boldsymbol{E}_{q}^{n}\right)+\beta k^{2}\left(\nabla E_{p}^{n}, \nabla r^{n}\right) \\
& \leq \frac{\nu k}{4}\left\|\nabla \tilde{\boldsymbol{\varepsilon}}^{n+\frac{1}{2}}\right\|^{2}+C k\left\|\boldsymbol{E}_{r}^{n}\right\|_{-1}^{2}+k\left\|\nabla E_{p}^{n}\right\|^{2}+C k^{3}\left\|\nabla r^{n}\right\|^{2} .
\end{aligned}
$$

Once again, we infer from (2.9) that

$$
\begin{aligned}
\beta k^{2}\left(\nabla\left(r^{n+1}-r^{n-1}\right), \nabla r^{n}\right)= & \frac{\beta k^{2}}{2}\left\{\left\|\nabla r^{n+1}\right\|^{2}-\left\|\nabla r^{n-1}\right\|^{2}\right. \\
& \left.+\left\|\nabla\left(r^{n}-r^{n-1}\right)\right\|^{2}-\left\|\nabla\left(r^{n+1}-r^{n}\right)\right\|^{2}\right\} .
\end{aligned}
$$

The nonlinear term can be handled as follows. We derive from (2.20) that

$$
\begin{aligned}
\boldsymbol{E}_{q}^{n}=\boldsymbol{Q}^{n}-\boldsymbol{Q}^{n-1}= & -\tilde{B}\left(\tilde{\boldsymbol{w}}^{n+\frac{1}{2}}, \tilde{\boldsymbol{e}}^{n+\frac{1}{2}}\right)-\tilde{B}\left(\boldsymbol{u}^{n-\frac{1}{2}}, \tilde{\boldsymbol{\varepsilon}}^{n+\frac{1}{2}}\right) \\
& -\tilde{B}\left(\tilde{\boldsymbol{\varepsilon}}^{n+\frac{1}{2}}, \tilde{\boldsymbol{u}}\left(t_{n+\frac{1}{2}}\right)\right)-\tilde{B}\left(\boldsymbol{e}^{n-\frac{1}{2}}, \boldsymbol{u}\left(t_{n+\frac{1}{2}}\right)-\boldsymbol{u}\left(t_{n-\frac{1}{2}}\right)\right) .
\end{aligned}
$$

Thanks to (2.3), we have

$$
\begin{aligned}
\left(\tilde{\boldsymbol{\varepsilon}}^{n+\frac{1}{2}}, \boldsymbol{E}_{q}^{n}\right)= & -\tilde{b}\left(\tilde{\boldsymbol{w}}^{n+\frac{1}{2}}, \tilde{\boldsymbol{e}}^{n+\frac{1}{2}}, \tilde{\boldsymbol{\varepsilon}}^{n+\frac{1}{2}}\right)-\tilde{b}\left(\tilde{\boldsymbol{\varepsilon}}^{n+\frac{1}{2}}, \tilde{\boldsymbol{u}}\left(t_{n+\frac{1}{2}}\right), \tilde{\boldsymbol{\varepsilon}}^{n+\frac{1}{2}}\right) \\
& -\tilde{b}\left(\boldsymbol{e}^{n-\frac{1}{2}}, \boldsymbol{u}\left(t_{n+\frac{1}{2}}\right)-\boldsymbol{u}\left(t_{n-\frac{1}{2}}\right), \tilde{\boldsymbol{\varepsilon}}^{n+\frac{1}{2}}\right) .
\end{aligned}
$$

Let $\boldsymbol{u}\left(t_{n+\frac{1}{2}}\right)-\boldsymbol{u}\left(t_{n-\frac{1}{2}}\right)=k \boldsymbol{u}_{t}\left(t_{n}^{\prime}\right)$; by using (2.6) and (2.4), we derive

$$
\begin{aligned}
k \tilde{b}\left(\tilde{\boldsymbol{w}}^{n+\frac{1}{2}}, \tilde{\boldsymbol{e}}^{n+\frac{1}{2}}, \tilde{\boldsymbol{\varepsilon}}^{n+\frac{1}{2}}\right)= & k \tilde{b}\left(\left(u\left(t_{n+\frac{1}{2}}\right)-u_{t}\left(t_{n-\frac{1}{2}}\right)\right)-\tilde{\boldsymbol{\varepsilon}}^{n+\frac{1}{2}}, \tilde{\boldsymbol{e}}^{n+\frac{1}{2}}, \tilde{\boldsymbol{\varepsilon}}^{n+\frac{1}{2}}\right) \\
\leq & C k^{2}\left\|\boldsymbol{u}_{t}\left(t_{n}^{\prime}\right)\right\|_{1}\left\|\tilde{\boldsymbol{e}}^{n+\frac{1}{2}}\right\|_{1}\left\|\tilde{\boldsymbol{\varepsilon}}^{n+\frac{1}{2}}\right\|_{1} \\
& +C k\left\|\tilde{\boldsymbol{e}}^{n+\frac{1}{2}}\right\|_{1}\left\|\tilde{\boldsymbol{\varepsilon}}^{n+\frac{1}{2}}\right\|^{\frac{1}{2}}\left\|\tilde{\boldsymbol{\varepsilon}}^{n+\frac{1}{2}}\right\|_{1}^{\frac{3}{2}}
\end{aligned}
$$

(thanks to (2.16) and Lemma 2.3)

$$
\leq \frac{\nu k}{4}\left\|\nabla \tilde{\boldsymbol{\varepsilon}}^{n+\frac{1}{2}}\right\|^{2}+C k^{3}\left\|\nabla \tilde{\boldsymbol{e}}^{n+\frac{1}{2}}\right\|^{2}+C k\left\|\tilde{\boldsymbol{\varepsilon}}^{n+\frac{1}{2}}\right\|^{2} .
$$


Similarly, by using (2.6) and (2.14)-(2.16), we obtain

$$
\begin{aligned}
k \tilde{b}\left(\boldsymbol{e}^{n-\frac{1}{2}}, \boldsymbol{u}\left(t_{n+\frac{1}{2}}\right)-\boldsymbol{u}\left(t_{n-\frac{1}{2}}\right), \tilde{\boldsymbol{\varepsilon}}^{n+\frac{1}{2}}\right) & =k^{2} \tilde{b}\left(\boldsymbol{e}^{n-\frac{1}{2}}, \boldsymbol{u}_{t}\left(t_{n}^{\prime}\right), \tilde{\boldsymbol{\varepsilon}}^{n+\frac{1}{2}}\right) \\
& \leq C k^{2}\left\|\boldsymbol{u}_{t}\left(t_{n}^{\prime}\right)\right\|_{1}\left\|\tilde{\boldsymbol{e}}^{n-\frac{1}{2}}\right\|\left\|_{1}\right\| \tilde{\boldsymbol{\varepsilon}}^{n+\frac{1}{2}} \|_{1} \\
& \leq \frac{\nu k}{4}\left\|\nabla \tilde{\boldsymbol{\varepsilon}}^{n+\frac{1}{2}}\right\|^{2}+C k^{3}\left\|\nabla \tilde{\boldsymbol{e}}^{n-\frac{1}{2}}\right\|^{2}, \\
k \tilde{b}\left(\tilde{\boldsymbol{\varepsilon}}^{n+\frac{1}{2}}, \tilde{\boldsymbol{u}}\left(t_{n+\frac{1}{2}}\right), \tilde{\boldsymbol{\varepsilon}}^{n+\frac{1}{2}}\right) & \leq C k\left\|\boldsymbol{u}\left(t_{n+\frac{1}{2}}\right)\right\|_{2}\left\|\tilde{\boldsymbol{\varepsilon}}^{n+\frac{1}{2}}\right\|\left\|\tilde{\boldsymbol{\varepsilon}}^{n+\frac{1}{2}}\right\|_{1} \\
& \leq \frac{\nu k}{4}\left\|\nabla \tilde{\boldsymbol{\varepsilon}}^{n+\frac{1}{2}}\right\|^{2}+C k\left\|\tilde{\boldsymbol{\varepsilon}}^{n+\frac{1}{2}}\right\|^{2} .
\end{aligned}
$$

Taking the sum of (2.32) for $n=2$ to $m$ and using the above relations, we arrive at

$$
\begin{aligned}
\left\|\varepsilon^{m+1}\right\|^{2} & +\nu k \sum_{n=2}^{m}\left\|\nabla \tilde{\boldsymbol{\varepsilon}}^{n+1}\right\|^{2}+\frac{\beta k^{2}}{2}\left(\left\|\nabla r^{m+1}\right\|^{2}+\left\|\nabla r^{m}\right\|^{2}\right) \\
\leq & \left\|\varepsilon^{2}\right\|^{2}+\frac{\beta k^{2}}{2}\left(\left\|\nabla r^{2}\right\|^{2}+\left\|\nabla r^{1}\right\|^{2}+\left\|\nabla\left(r^{m+1}-r^{m}\right)\right\|^{2}\right) \\
& +C k \sum_{n=2}^{m}\left\{\left\|\tilde{\boldsymbol{\varepsilon}}^{n+\frac{1}{2}}\right\|^{2}+k^{2}\left(\left\|\tilde{\boldsymbol{e}}^{n+\frac{1}{2}}\right\|_{1}^{2}+\tilde{\boldsymbol{e}}^{n-\frac{1}{2}} \|_{1}^{2}\right)\right\} \\
& +C k \sum_{n=2}^{m}\left\{\left\|\boldsymbol{E}_{r}^{n}\right\|_{-1}^{2}+\left\|\nabla E_{p}^{n}\right\|^{2}\right\}+C k^{3} \sum_{n=2}^{m}\left(\left\|\nabla r^{n}\right\|^{2}+\left\|\nabla r^{n-1}\right\|^{2}\right) .
\end{aligned}
$$

Similarly to (2.25), we have

$$
\begin{aligned}
\frac{\beta k^{2}}{2}\left\|\nabla\left(r^{m+1}-r^{m}\right)\right\|^{2} \leq & \frac{1+\frac{\delta}{2}}{1+\delta}\left\|\varepsilon^{m+1}\right\|^{2}+C k^{4}\left\|p_{t}\right\|_{C\left(\left[t_{0}, T_{1}\right] ; H^{1}(\Omega)\right)}^{2} \\
& +\frac{1-\frac{\delta}{2}}{2} \beta k^{2}\left(\left\|\nabla r^{m+1}\right\|^{2}+\left\|\nabla r^{m}\right\|^{2}\right) .
\end{aligned}
$$

We derive from Lemma A2 that

$$
\left\|\varepsilon^{2}\right\|^{2}=\left\|e^{2}-e^{1}\right\|^{2} \leq 2\left(\left\|e^{2}\right\|^{2}+\left\|e^{1}\right\|^{2}\right) \leq C k^{4} .
$$

Taking the inner product of (2.18) with $q^{n+1}-q^{n}$ for $n=0$, 1 , we find

$$
\left\|\nabla\left(q^{n+1}-q^{n}\right)\right\| \leq C\left(\frac{1}{k}\left\|e^{n+1}\right\|+\left\|\nabla\left(p\left(t_{n+1}\right)-p\left(t_{n}\right)\right)\right\|\right) \leq C k, \quad n=0,1 .
$$

Taking into account the above estimates in (2.34) and using (2.26) and Lemma A1, we obtain

$$
\begin{aligned}
\frac{\delta}{2(\delta+1)}\left\|\varepsilon^{m+1}\right\|^{2} & +\frac{\nu k}{4} \sum_{n=2}^{m}\left\|\nabla\left(\varepsilon^{n+1}+\varepsilon^{n}\right)\right\|^{2}+\delta \beta k^{2}\left(\left\|\nabla r^{m+1}\right\|^{2}+\left\|\nabla r^{m}\right\|^{2}\right) \\
& \leq C k^{4}+C k \sum_{n=2}^{m}\left(\left\|\varepsilon^{n+1}\right\|^{2}+k^{2}\left\|\nabla r^{n}\right\|^{2}\right)
\end{aligned}
$$

Applying Lemma 2.1 to the above inequality, we conclude that

$$
\begin{aligned}
& \left\|\varepsilon^{m+1}\right\|^{2}+k \sum_{n=2}^{m}\left\|\nabla\left(\varepsilon^{n+1}+\varepsilon^{n}\right)\right\|^{2} \\
& \quad+k^{2}\left(\left\|\nabla r^{m+1}\right\|^{2}+\left\|\nabla r^{m}\right\|^{2}\right) \leq C k^{4}, \forall 2 \leq m \leq M-1,
\end{aligned}
$$

which completes the proof of Lemma 2.4. 


\section{ERror estimates}

The main result in this section is collected in the following theorem.

Theorem 3.1. Assume (2.13) and (2.15). Given $t_{0} \in\left(0, T_{1}\right), \beta>\frac{1}{4}$ and $\left(\boldsymbol{u}^{0}, p^{0}\right)$ satisfying (2.12), there exists a positive constant $C$ depending on the data and $t_{0}$ such that

$$
\begin{aligned}
k \sum_{n=1}^{m}\left\|\boldsymbol{u}\left(t_{n}\right)-\boldsymbol{u}^{n}\right\|^{2}+ & k^{2}\left\|\nabla\left(\boldsymbol{u}\left(t_{m}\right)-\boldsymbol{u}^{m}\right)\right\|^{2} \\
& +k^{2}\left\|p\left(t_{m}\right)-p^{m}\right\|^{2} \leq C k^{4}, \forall 1 \leq m \leq M=\left[\frac{T_{1}-t_{0}}{k}\right],
\end{aligned}
$$

where $(\boldsymbol{u}(t), p(t))$ and $\left(\boldsymbol{u}^{n}, p^{n}\right)$ are respectively the solutions of (1.1)-(1.2) and of (1.10)-(1.11).

The remainder of this section is devoted to prove this theorem. We will introduce an auxiliary linear problem and split the error into two parts. The first part is associated with the time-dependent linear Stokes operator and the second part is associated with the nonlinear term. It will become clear that the dominating error term is introduced by the approximation of the linear operator (see Lemmas 3.1 and 3.2 ) while the approximation error associated with the nonlinear term is relatively small and easy to handle (see Lemma 3.3).

3.1. Error estimates for a linear auxiliary problem. We define $\left(\boldsymbol{v}^{n+1}, r^{n+1}\right)$ to be the solution of the following auxiliary linear problem:

$$
\left\{\begin{array}{l}
\frac{\boldsymbol{v}^{n+1}-\boldsymbol{v}^{n}}{k}-\frac{\nu}{2} \Delta\left(\boldsymbol{v}^{n+1}+\boldsymbol{v}^{n}\right)+\nabla r^{n}=\boldsymbol{f}\left(t_{n+\frac{1}{2}}\right)-\tilde{B}\left(\tilde{\boldsymbol{u}}\left(t_{n+\frac{1}{2}}\right), \tilde{\boldsymbol{u}}\left(t_{n+\frac{1}{2}}\right)\right) \\
\left.\boldsymbol{v}^{n+1}\right|_{\partial \Omega}=0
\end{array}\right.
$$

$$
\nabla \boldsymbol{v}^{n+1}-\beta k \Delta\left(r^{n+1}-r^{n}\right)=0,\left.\quad \frac{\partial r^{n+1}}{\partial \boldsymbol{n}}\right|_{\partial \Omega}=\left.\frac{\partial r^{n}}{\partial \boldsymbol{n}}\right|_{\partial \Omega}
$$

with $\left(\boldsymbol{v}^{0}, r^{0}\right)=\left(\boldsymbol{u}^{0}, p^{0}\right)$. We will also use the following relation derived from (3.2):

$$
\nabla\left(\boldsymbol{v}^{n+1}+\boldsymbol{v}^{n}\right)-\beta k \Delta\left(r^{n+1}-r^{n-1}\right)=0,\left.\quad \frac{\partial r^{n+1}}{\partial \boldsymbol{n}}\right|_{\partial \Omega}=\left.\frac{\partial r^{n-1}}{\partial \boldsymbol{n}}\right|_{\partial \Omega}
$$

Denoting $\boldsymbol{\xi}^{n}=\boldsymbol{u}\left(t_{n}\right)-\boldsymbol{v}^{n}$ and $\phi^{n}=p\left(t_{n}\right)-r^{n}$, it is obvious that the results in Lemmas 2.3 and 2.4 for (1.10)-(1.11) are also valid for this auxiliary linear system. Namely, we have

$$
\left\|\frac{\boldsymbol{\xi}^{m+1}-\boldsymbol{\xi}^{m}}{k}\right\|^{2}+\left\|r^{m+1}-r^{m}\right\|_{1}^{2}+\left\|\phi^{m+1}-\phi^{m}\right\|_{1}^{2} \leq C k^{2}, \forall 1 \leq m \leq M-1
$$


Lemma 3.1. Under the assumptions of Theorem 3.1, we have

$$
k \sum_{n=1}^{m}\left\|\boldsymbol{u}\left(t_{n}\right)-\boldsymbol{v}^{n}\right\|^{2} \leq C k^{4}, \forall 1 \leq m \leq M .
$$

Proof. Subtracting (3.1)-(3.3) from (1.1)-(1.2), we obtain the error equations

$$
\begin{gathered}
\frac{\boldsymbol{\xi}^{n+1}-\boldsymbol{\xi}^{n}}{k}-\frac{\nu}{2} \Delta\left(\boldsymbol{\xi}^{n+1}+\boldsymbol{\xi}^{n}\right)+\nabla \phi^{n}=\boldsymbol{R}^{n}, \\
\nabla\left(\boldsymbol{\xi}^{n+1}+\boldsymbol{\xi}^{n}\right)+\beta k \Delta\left(r^{n+1}-r^{n-1}\right)=0,\left.\quad \frac{\partial r^{n+1}}{\partial \boldsymbol{n}}\right|_{\partial \Omega}=\left.\frac{\partial r^{n-1}}{\partial \boldsymbol{n}}\right|_{\partial \Omega} .
\end{gathered}
$$

We note that $\boldsymbol{R}^{n}$ is the truncation error defined in (2.21).

For any $0 \leq N \leq M-1$, we define $\left(\boldsymbol{w}^{n}, q^{n}\right)$ to be the solution of the time-reversed discrete parabolic duality problem:

$$
\begin{aligned}
& \frac{\boldsymbol{w}^{n+1}-\boldsymbol{w}^{n}}{k}+\frac{\nu}{2} \Delta\left(\boldsymbol{w}^{n+1}+\boldsymbol{w}^{n}\right)+\nabla q^{n}=\boldsymbol{\xi}^{n+1}+\boldsymbol{\xi}^{n}, \\
& \nabla \boldsymbol{w}^{n}=0,\left.\quad \boldsymbol{w}^{n}\right|_{\partial \Omega}=0,
\end{aligned}
$$

for $n=N, N-1, \ldots, 0$, with the "initial" condition $\boldsymbol{w}^{N+1}=0$. By successively taking the inner product of (3.8) with $\Delta\left(\boldsymbol{w}^{n+1}+\boldsymbol{w}^{n}\right)$ and with $\boldsymbol{w}^{n+1}-\boldsymbol{w}^{n}$, we can derive by a standard procedure that

$$
\begin{aligned}
&\left\|\boldsymbol{w}^{m}\right\|_{1}^{2}+k \sum_{n=0}^{m}\left(\left\|\boldsymbol{w}^{n+1}+\boldsymbol{w}^{n}\right\|_{2}^{2}+\left\|q^{n}\right\|_{1}^{2}\right) \\
& \leq C k \sum_{n=0}^{m}\left\|\boldsymbol{\xi}^{n+1}+\boldsymbol{\xi}^{n}\right\|^{2}, \forall 0 \leq m \leq N .
\end{aligned}
$$

We now take the inner product of (3.8) with $\boldsymbol{\xi}^{n+1}+\boldsymbol{\xi}^{n}$ to obtain

$$
\begin{aligned}
\left\|\boldsymbol{\xi}^{n+1}+\boldsymbol{\xi}^{n}\right\|^{2}= & \frac{1}{k}\left(\boldsymbol{\xi}^{n+1}+\boldsymbol{\xi}^{n}, \boldsymbol{w}^{n+1}-\boldsymbol{w}^{n}\right) \\
& +\frac{\nu}{2}\left(\Delta\left(\boldsymbol{\xi}^{n+1}+\boldsymbol{\xi}^{n}\right), \boldsymbol{w}^{n+1}+\boldsymbol{w}^{n}\right)-\left(q^{n}, \nabla\left(\boldsymbol{\xi}^{n+1}+\boldsymbol{\xi}^{n}\right)\right) .
\end{aligned}
$$

Using the identity

$$
\begin{aligned}
\frac{1}{k}\left(\boldsymbol{\xi}^{n+1}+\boldsymbol{\xi}^{n}, \boldsymbol{w}^{n+1}-\boldsymbol{w}^{n}\right)=\frac{2}{k}\left[\left(\boldsymbol{\xi}^{n+1}, \boldsymbol{w}^{n+1}\right)-\right. & \left.\left(\boldsymbol{\xi}^{n}, \boldsymbol{w}^{n}\right)\right] \\
& -\frac{1}{k}\left(\boldsymbol{\xi}^{n+1}-\boldsymbol{\xi}^{n}, \boldsymbol{w}^{n+1}+\boldsymbol{w}^{n}\right),
\end{aligned}
$$

and taking into account (3.6)-(3.7) and (3.9), we derive from (3.11) that

$$
\begin{aligned}
k\left\|\boldsymbol{\xi}^{n+1}+\boldsymbol{\xi}^{n}\right\|^{2}=2\left[\left(\boldsymbol{\xi}^{n+1},\right.\right. & \left.\left.\boldsymbol{w}^{n+1}\right)-\left(\boldsymbol{\xi}^{n}, \boldsymbol{w}^{n}\right)\right] \\
& -\beta k^{2}\left(\nabla q^{n}, \nabla\left(r^{n+1}-r^{n-1}\right)\right)+k\left(\boldsymbol{R}^{n}, \boldsymbol{w}^{n+1}+\boldsymbol{w}^{n}\right) .
\end{aligned}
$$


Summing up the last relation for $n$ from 1 to $N$, since $\boldsymbol{w}^{N+1}=0$ and $\left(\boldsymbol{R}_{2}^{n}, \boldsymbol{w}^{n+1}+\right.$ $\left.\boldsymbol{w}^{n}\right)=0$, we derive

$$
\begin{aligned}
k \sum_{n=1}^{N}\left\|\boldsymbol{\xi}^{n+1}+\boldsymbol{\xi}^{n}\right\|^{2}= & -2\left(\boldsymbol{\xi}^{1}, \boldsymbol{w}^{1}\right) \\
& -\sum_{n=1}^{N}\left\{\beta k^{2}\left(\nabla q^{n}, \nabla\left(r^{n+1}-r^{n-1}\right)\right)+k\left(\boldsymbol{R}_{1}^{n}, \boldsymbol{w}^{n+1}+\boldsymbol{w}^{n}\right)\right\} \\
\leq \delta\left\|\boldsymbol{w}^{1}\right\|^{2}+C\left\|\boldsymbol{\xi}^{1}\right\|^{2}+\delta k \sum_{n=1}^{N}\left(\left\|q^{n}\right\|_{1}^{2}+\left\|\boldsymbol{w}^{n+1}+\boldsymbol{w}^{n}\right\|_{1}^{2}\right) & \\
& +C k \sum_{n=1}^{N}\left(k^{2}\left\|r^{n+1}-r^{n-1}\right\|_{1}^{2}+\left\|\boldsymbol{R}_{1}^{n}\right\|_{-1}^{2}\right) .
\end{aligned}
$$

From Lemma A2 (which holds also for the linear auxiliary problem), we have $\left\|\xi^{1}\right\| \leq$ $C k^{2}$. Then, choosing $\delta$ sufficiently small, thanks to (3.10), (3.5) and Lemma A1, we derive

$$
k \sum_{n=1}^{N}\left\|\boldsymbol{\xi}^{n+1}+\boldsymbol{\xi}^{n}\right\|^{2} \leq C k \sum_{n=1}^{N}\left(k^{2}\left\|r^{n+1}-r^{n-1}\right\|_{1}^{2}+\left\|\boldsymbol{R}_{1}^{n}\right\|_{-1}^{2}\right)+C\left\|\boldsymbol{\xi}^{1}\right\|^{2} \leq C k^{4} .
$$

Writing $2 \boldsymbol{\xi}^{n+1}=\left(\boldsymbol{\xi}^{n+1}+\boldsymbol{\xi}^{n}\right)+\left(\boldsymbol{\xi}^{n+1}-\boldsymbol{\xi}^{n}\right)$, we derive from (3.5) and the above inequality that

$$
2 k \sum_{n=1}^{M-1}\left\|\boldsymbol{\xi}^{n+1}\right\|^{2} \leq k \sum_{n=1}^{M-1}\left(\left\|\boldsymbol{\xi}^{n+1}+\boldsymbol{\xi}^{n}\right\|^{2}+\left\|\boldsymbol{\xi}^{n+1}-\boldsymbol{\xi}^{n}\right\|^{2}\right) \leq C k^{4} .
$$

Lemma 3.2. Under the assumptions of Theorem 3.1, we have

$$
\left\|\boldsymbol{u}\left(t_{n}\right)-\boldsymbol{v}^{n}\right\|_{1}^{2}+\left\|p\left(t_{n}\right)-r^{n}\right\|^{2} \leq C k^{2}, \forall 0 \leq n \leq M .
$$

Proof. Taking the inner product of (3.6) with $\left(\boldsymbol{\xi}^{n+1}-\boldsymbol{\xi}^{n}\right)$, we obtain

$$
\begin{aligned}
\frac{1}{k}\left\|\boldsymbol{\xi}^{n+1}-\boldsymbol{\xi}^{n}\right\|^{2} & +\frac{\nu}{2}\left(\left\|\nabla \boldsymbol{\xi}^{n+1}\right\|^{2}-\left\|\nabla \boldsymbol{\xi}^{n}\right\|^{2}\right) \\
& =\left(\boldsymbol{R}^{n}, \boldsymbol{\xi}^{n+1}-\boldsymbol{\xi}^{n}\right)+\left(\phi^{n}, \nabla\left(\boldsymbol{\xi}^{n+1}-\boldsymbol{\xi}^{n}\right)\right) \\
& \leq \frac{1}{2 k}\left\|\boldsymbol{\xi}^{n+1}-\boldsymbol{\xi}^{n}\right\|^{2}+\frac{k}{2}\left\|\boldsymbol{R}^{n}\right\|^{2}+\left(\phi^{n}, \nabla\left(\boldsymbol{\xi}^{n+1}-\boldsymbol{\xi}^{n}\right)\right) .
\end{aligned}
$$

We derive from (3.2) that

$$
\left(\nabla\left(\boldsymbol{\xi}^{n+1}-\boldsymbol{\xi}^{n}\right), \gamma\right)-\beta k\left(\nabla\left(r^{n+1}-2 r^{n}+r^{n-1}\right), \nabla \gamma\right)=0, \forall \gamma \in H^{1}(\Omega) / \mathbb{R} .
$$

Therefore,

$$
\begin{aligned}
&\left(\phi^{n}, \nabla\left(\boldsymbol{\xi}^{n+1}-\boldsymbol{\xi}^{n}\right)\right)=\beta k\left(\nabla \phi^{n}, \nabla\left(r^{n+1}-2 r^{n}+r^{n-1}\right)\right) \\
&= \beta k\left(\left(\nabla \phi^{n}, \nabla\left(r^{n+1}-r^{n}\right)\right)-\left(\nabla \phi^{n-1}, \nabla\left(r^{n}-r^{n-1}\right)\right)\right) \\
&-\beta k\left(\nabla\left(\phi^{n}-\phi^{n-1}\right), \nabla\left(r^{n}-r^{n-1}\right)\right) .
\end{aligned}
$$


In view of (3.5), we get

$$
\begin{aligned}
& \left\|\nabla \phi^{m}\right\|^{2}=\left\|\nabla \sum_{n=1}^{m}\left(\phi^{n}-\phi^{n-1}\right)+\nabla \phi^{0}\right\|^{2} \\
& \quad \leq 2 m \sum_{n=1}^{m}\left\|\nabla\left(\phi^{n}-\phi^{n-1}\right)\right\|^{2}+2\left\|\nabla \phi^{0}\right\|^{2} \leq C, \quad \forall 1 \leq m \leq M .
\end{aligned}
$$

Summing up (3.13), thanks to the above inequality and (3.5), we obtain that for any $1 \leq m \leq M-1$

$$
\begin{aligned}
\sum_{n=1}^{m}\left(\phi^{n}, \nabla\left(\boldsymbol{\xi}^{n+1}-\boldsymbol{\xi}^{n}\right)\right)= & \beta k\left(\nabla \phi^{m}, \nabla\left(r^{m+1}-r^{m}\right)\right)-\beta k\left(\nabla \phi^{0}, \nabla\left(r^{1}-r^{0}\right)\right) \\
& -\beta k \sum_{n=1}^{m}\left(\left(\nabla\left(\phi^{n}-\phi^{n-1}\right), \nabla\left(r^{n}-r^{n-1}\right)\right)\right. \\
\leq \frac{\beta}{2}\left(k^{2}\left\|\nabla \phi^{m}\right\|^{2}+\right. & \left.\left\|\nabla\left(r^{m+1}-r^{m}\right)\right\|^{2}+k^{2}\left\|\nabla \phi^{0}\right\|^{2}+\left\|\nabla\left(r^{1}-r^{0}\right)\right\|^{2}\right) \\
+ & C k \sum_{n=1}^{m}\left\{\left\|\nabla\left(r^{n}-r^{n-1}\right)\right\|^{2}+\left\|\nabla\left(\phi^{n}-\phi^{n-1}\right)\right\|^{2}\right\} \leq C k^{2} .
\end{aligned}
$$

Taking the sum of (3.12) for $n$ from 1 to $m$ and collecting the above inequalities, thanks to (2.12) and Lemma A1 (Lemma A1 is certainly applicable to the linear auxiliary problem), we obtain

$$
\begin{aligned}
\frac{\nu}{2}\left\|\nabla \boldsymbol{\xi}^{m+1}\right\|^{2}+\frac{1}{2 k} \sum_{n=1}^{m} \| \boldsymbol{\xi}^{n+1} & -\boldsymbol{\xi}^{n} \|^{2} \\
& \leq C k \sum_{n=1}^{m}\left\|\boldsymbol{R}^{n}\right\|^{2}+C k^{2} \leq C k^{2}, \forall 1 \leq m \leq M-1 .
\end{aligned}
$$

Finally, we derive from (3.6), Lemma A1 and the above inequality that

$$
\begin{aligned}
\left\|\phi^{n}\right\| & \leq C\left(\left\|\frac{\boldsymbol{\xi}^{n+1}-\boldsymbol{\xi}^{n}}{k}\right\|_{-1}+\left\|\boldsymbol{R}^{n}\right\|+\left\|\boldsymbol{\xi}^{n+1}+\boldsymbol{\xi}^{n}\right\|_{1}\right) \\
& \leq C k, \forall 1 \leq n \leq M-1 . \quad \square
\end{aligned}
$$

3.2. Error estimates for the nonlinear problem. We denote $\boldsymbol{\eta}^{n}=\boldsymbol{v}^{n}-\boldsymbol{u}^{n}$ and $\psi^{n}=r^{n}-p^{n}$. Subtracting (1.10)-(1.11) from (3.1)-(3.3), we obtain

$$
\frac{\boldsymbol{\eta}^{n+1}-\boldsymbol{\eta}^{n}}{k}-\frac{\nu}{2} \Delta\left(\boldsymbol{\eta}^{n+1}+\boldsymbol{\eta}^{n}\right)+\nabla \psi^{n}=-\boldsymbol{Q}^{n}
$$

$$
\nabla\left(\boldsymbol{\eta}^{n+1}+\boldsymbol{\eta}^{n}\right)-\beta k \Delta\left(\psi^{n+1}-\psi^{n-1}\right)=0,\left.\quad \frac{\partial \psi^{n+1}}{\partial \boldsymbol{n}}\right|_{\partial \Omega}=\left.\frac{\partial \psi^{n-1}}{\partial \boldsymbol{n}}\right|_{\partial \Omega},
$$

with $\boldsymbol{\eta}^{0}=0$ and $\psi^{0}=0$. We recall that $\boldsymbol{Q}^{n}$ is defined in (2.20). 
Lemma 3.3. Under the assumptions of Theorem 3.1, we have

$$
\left\|\boldsymbol{\eta}^{m+1}\right\|^{2}+k \sum_{n=1}^{m}\left\|\nabla\left(\boldsymbol{\eta}^{n+1}+\boldsymbol{\eta}^{n}\right)\right\|^{2}+k^{2}\left\|\nabla \psi^{m+1}\right\|^{2} \leq C k^{4}, \forall 1 \leq m \leq M-1
$$

Proof. We note that $\boldsymbol{u}\left(t_{n}\right)-\boldsymbol{u}^{n}=\boldsymbol{e}^{n}=\boldsymbol{\xi}^{n}+\boldsymbol{\eta}^{n}$. Therefore,

$$
\begin{aligned}
\boldsymbol{Q}^{n} & =\tilde{B}\left(\tilde{\boldsymbol{u}}\left(t_{n+\frac{1}{2}}\right), \tilde{\boldsymbol{u}}\left(t_{n+\frac{1}{2}}\right)\right)-\tilde{B}\left(\tilde{\boldsymbol{u}}^{n+\frac{1}{2}}, \tilde{\boldsymbol{u}}^{n+\frac{1}{2}}\right) \\
& =\tilde{B}\left(\tilde{\boldsymbol{u}}^{n+\frac{1}{2}}, \tilde{\boldsymbol{e}}^{n+\frac{1}{2}}\right)+\tilde{B}\left(\tilde{\boldsymbol{e}}^{n+\frac{1}{2}}, \tilde{\boldsymbol{u}}\left(t_{n+\frac{1}{2}}\right)\right) \\
& =\tilde{B}\left(\tilde{\boldsymbol{u}}^{n+\frac{1}{2}}, \tilde{\boldsymbol{\xi}}^{n+\frac{1}{2}}+\tilde{\boldsymbol{\eta}}^{n+\frac{1}{2}}\right)+\tilde{B}\left(\tilde{\boldsymbol{\xi}}^{n+\frac{1}{2}}+\tilde{\boldsymbol{\eta}}^{n+\frac{1}{2}}, \tilde{\boldsymbol{u}}\left(t_{n+\frac{1}{2}}\right)\right) .
\end{aligned}
$$

Taking the inner product of (3.14) with $k \tilde{\boldsymbol{\eta}}^{n+\frac{1}{2}}$ and of (3.15) with $k\left(3 \psi^{n}-\psi^{n-1}\right)$, and summing up the two relations for $n=1$ to $m$, similarly as in the proof of Lemma 2.2, we obtain

$$
\begin{aligned}
\left\|\boldsymbol{\eta}^{m+1}\right\|^{2} & +\nu k \sum_{n=1}^{m}\left\|\nabla \tilde{\boldsymbol{\eta}}^{n+\frac{1}{2}}\right\|^{2}+\beta k^{2}\left(\left\|\nabla \psi^{m+1}\right\|^{2}+\left\|\nabla \psi^{m}\right\|^{2}\right) \\
\leq & \left\|\boldsymbol{\eta}^{1}\right\|^{2}+\frac{\beta k^{2}}{2}\left(\left\|\nabla \psi^{1}\right\|^{2}+\left\|\nabla \psi^{0}\right\|^{2}+\left\|\nabla\left(\psi^{m+1}-\psi^{m}\right)\right\|^{2}\right) \\
& \quad k \sum_{n=1}^{m}\left\{\tilde{b}\left(\tilde{\boldsymbol{u}}^{n+\frac{1}{2}}, \tilde{\boldsymbol{\xi}}^{n+\frac{1}{2}}, \tilde{\boldsymbol{\eta}}^{n+\frac{1}{2}}\right)+\tilde{b}\left(\tilde{\boldsymbol{\xi}}^{n+\frac{1}{2}}+\tilde{\boldsymbol{\eta}}^{n+\frac{1}{2}}, \tilde{\boldsymbol{u}}\left(t_{n+\frac{1}{2}}\right), \tilde{\boldsymbol{\eta}}^{n+\frac{1}{2}}\right)\right\} .
\end{aligned}
$$

We now bound the nonlinear terms above. Thanks to Lemma 2.3, we derive by using (2.6) that

$$
\begin{aligned}
k \tilde{b}\left(\tilde{\boldsymbol{u}}^{n+\frac{1}{2}}, \tilde{\boldsymbol{\xi}}^{n+\frac{1}{2}}, \tilde{\boldsymbol{\eta}}^{n+\frac{1}{2}}\right) & \leq C k\left\|\tilde{\boldsymbol{u}}^{n+\frac{1}{2}}\right\|\left\|_{2}\right\| \tilde{\boldsymbol{\xi}}^{n+\frac{1}{2}}\|\| \tilde{\boldsymbol{\eta}}^{n+\frac{1}{2}} \|_{1} \\
& \leq \frac{\nu k}{4}\left\|\nabla \tilde{\boldsymbol{\eta}}^{n+\frac{1}{2}}\right\|^{2}+C k\left\|\tilde{\boldsymbol{\xi}}^{n+\frac{1}{2}}\right\|^{2} .
\end{aligned}
$$

Thanks to (2.6) and (2.14), we have

$$
\begin{aligned}
k \tilde{b}\left(\tilde{\boldsymbol{\xi}}^{n+\frac{1}{2}}+\tilde{\boldsymbol{\eta}}^{n+\frac{1}{2}}, \tilde{\boldsymbol{u}}\left(t_{n+\frac{1}{2}}\right), \tilde{\boldsymbol{\eta}}^{n+\frac{1}{2}}\right) & \leq C k\left\|\tilde{\boldsymbol{\xi}}^{n+\frac{1}{2}}+\tilde{\boldsymbol{\eta}}^{n+\frac{1}{2}}\right\|\left\|\tilde{\boldsymbol{u}}\left(t_{n+\frac{1}{2}}\right)\right\|_{2}\left\|\tilde{\boldsymbol{\eta}}^{n+\frac{1}{2}}\right\|_{1} \\
& \leq \frac{\nu k}{4}\left\|\nabla \tilde{\boldsymbol{\eta}}^{n+\frac{1}{2}}\right\|^{2}+C k\left(\left\|\tilde{\boldsymbol{\xi}}^{n+\frac{1}{2}}\right\|^{2}+\left\|\tilde{\boldsymbol{\eta}}^{n+\frac{1}{2}}\right\|^{2}\right) .
\end{aligned}
$$

As in the proof of Lemma 2.4, for $\delta=\beta-\frac{1}{4}$, we have

$$
\frac{\beta k^{2}}{2}\left\|\nabla\left(\psi^{m+1}-\psi^{m}\right)\right\|^{2} \leq \frac{1+\frac{\delta}{2}}{1+\delta}\|\boldsymbol{\eta}\|^{2}+\frac{1-\frac{\delta}{2}}{2} \beta k^{2}\left(\left\|\nabla \psi^{m+1}\right\|^{2}+\left\|\nabla \psi^{m}\right\|^{2}\right) .
$$

Since $\boldsymbol{\eta}^{0}=0$ and $\psi^{0}=0$, we can easily (as in Lemma A2) prove that

$$
\left\|\boldsymbol{\eta}^{1}\right\|^{2}+k^{2}\left\|\nabla \psi^{1}\right\|^{2} \leq C k^{4}
$$


Collecting the above inequalities into (3.17) and using (2.12) and Lemma 3.1, we arrive at

$$
\begin{gathered}
\frac{\delta}{2(\delta+1)}\left\|\boldsymbol{\eta}^{m+1}\right\|^{2}+\frac{\nu k}{2} \sum_{n=1}^{m}\left\|\nabla \tilde{\boldsymbol{\eta}}^{n+\frac{1}{2}}\right\|^{2}+\frac{\delta \beta k^{2}}{4}\left(\left\|\nabla \psi^{m+1}\right\|^{2}+\left\|\nabla \psi^{m}\right\|^{2}\right) \\
\leq C k^{4}+C k \sum_{n=1}^{m}\left\|\boldsymbol{\eta}^{n+1}\right\|^{2}, \forall 1 \leq m \leq M-1 .
\end{gathered}
$$

We conclude the proof by applying Lemma 2.1 to the above inequality.

Proof of Theorem 3.1. We note that

$$
\boldsymbol{u}\left(t_{n}\right)-u^{n}=\boldsymbol{\xi}^{n}+\boldsymbol{\eta}^{n}, \quad p\left(t_{n}\right)-p^{n}=\phi^{n}+\psi^{n} .
$$

In view of Lemmas 3.1-3.3, the result in Theorem 3.1 holds provided that the following additional crude estimate can be established:

$$
\left\|\nabla \boldsymbol{\eta}^{n}\right\|^{2} \leq C k^{2}, \forall 0 \leq n \leq M .
$$

In order to derive (3.18), we take the inner product of (3.14) with $\left(\boldsymbol{\eta}^{n+1}-\boldsymbol{\eta}^{n}\right)$ to obtain

$$
\begin{aligned}
\frac{1}{k}\left\|\boldsymbol{\eta}^{n+1}-\boldsymbol{\eta}^{n}\right\|^{2}+ & \frac{\nu}{2}\left(\left\|\nabla \boldsymbol{\eta}^{n+1}\right\|^{2}-\left\|\nabla \boldsymbol{\eta}^{n}\right\|^{2}\right)=-\left(\nabla \psi^{n}+\boldsymbol{Q}^{n}, \boldsymbol{\eta}^{n+1}-\boldsymbol{\eta}^{n}\right) \\
& \leq \frac{1}{2 k}\left\|\boldsymbol{\eta}^{n+1}-\boldsymbol{\eta}^{n}\right\|^{2}+\frac{k}{2}\left\|\nabla \psi^{n}\right\|^{2}-\left(\boldsymbol{Q}^{n}, \boldsymbol{\eta}^{n+1}-\boldsymbol{\eta}^{n}\right)
\end{aligned}
$$

Thanks to Lemma 2.3 and (2.14), we derive from (3.16) and (2.6) that

$$
\begin{aligned}
& \left(\boldsymbol{Q}^{n}, \boldsymbol{\eta}^{n+1}-\boldsymbol{\eta}^{n}\right)=\tilde{b}\left(\tilde{\boldsymbol{u}}^{n+\frac{1}{2}}, \tilde{\boldsymbol{\xi}}^{n+\frac{1}{2}}+\tilde{\boldsymbol{\eta}}^{n+\frac{1}{2}}, \boldsymbol{\eta}^{n+1}-\boldsymbol{\eta}^{n}\right) \\
& +\tilde{b}\left(\tilde{\boldsymbol{\xi}}^{n+\frac{1}{2}}+\tilde{\boldsymbol{\eta}}^{n+\frac{1}{2}}, \tilde{\boldsymbol{u}}\left(t_{n+\frac{1}{2}}\right), \boldsymbol{\eta}^{n+1}-\boldsymbol{\eta}^{n}\right) \\
& \leq C\left(\left\|\tilde{\boldsymbol{u}}^{n+\frac{1}{2}}\right\|_{2}^{2}\left\|\tilde{\boldsymbol{\xi}}^{n+\frac{1}{2}}\right\|_{1}+\left\|\tilde{\boldsymbol{\xi}}^{n+\frac{1}{2}}+\tilde{\boldsymbol{\eta}}^{n+\frac{1}{2}}\right\|_{1}\left\|\tilde{\boldsymbol{u}}\left(t_{n+\frac{1}{2}}\right)\right\|_{2}\right)\left\|\boldsymbol{\eta}^{n+1}-\boldsymbol{\eta}^{n}\right\| \\
& \leq \frac{1}{2 k}\left\|\boldsymbol{\eta}^{n+1}-\boldsymbol{\eta}^{n}\right\|^{2}+C k\left(\left\|\tilde{\boldsymbol{\xi}}^{n+\frac{1}{2}}\right\|_{1}^{2}+\left\|\tilde{\boldsymbol{\eta}}^{n+\frac{1}{2}}\right\|_{1}^{2}\right) .
\end{aligned}
$$

Taking the sum of (3.19) for $n=1$ to $m$, thanks to Lemmas 3.2-3.3, we obtain (3.18).

\section{AnAlysis FOR The SCHEME (1.3)-(1.4)}

We can also prove a similar result for the scheme (1.3)-(1.4).

Theorem 4.1. We assume (2.13) and (2.15). Given $t_{0} \in\left(0, T_{1}\right)$ and $\left(\boldsymbol{u}^{0}, p^{0}\right)$ satisfying (2.12), there exists a positive constant $C$ depending on the data and $t_{0}$ such that

$$
\begin{aligned}
k \sum_{n=1}^{m}\left\|\boldsymbol{u}\left(t_{n}\right)-\tilde{\boldsymbol{u}}^{n}\right\|^{2} & +k^{2}\left\|\nabla\left(\boldsymbol{u}\left(t_{m}\right)-\tilde{\boldsymbol{u}}^{m}\right)\right\|^{2} \\
& +k^{2}\left\|p\left(t_{m}\right)-p^{m}\right\| \leq C k^{4}, \forall 1 \leq m \leq\left[\left(T_{1}-t_{0}\right) / k\right],
\end{aligned}
$$


JIE SHEN

$$
k \sum_{n=1}^{m}\left\|\boldsymbol{u}\left(t_{n}\right)-\boldsymbol{u}^{n}\right\|^{2}+k^{2}\left\|\nabla\left(\boldsymbol{u}\left(t_{m}\right)-\boldsymbol{u}^{m}\right)\right\|^{2} \leq C k^{4}, \forall 1 \leq m \leq\left[\frac{T_{1}-t_{0}}{k}\right]
$$

where $(\boldsymbol{u}(t), p(t))$ and $\left(\tilde{\boldsymbol{u}}^{n}, \boldsymbol{u}^{n}, p^{n}\right)$ are respectively the solutions of (1.1)-(1.2) and of (1.3)-(1.4).

Sketch of the proof. The proof of (4.1) is basically the same as that of Theorem 3.1. The result in (4.2) is a direct consequence of (4.1) and the inequality (cf. [22])

$$
\|P \boldsymbol{v}\|_{i} \leq C(\Omega)\|\boldsymbol{v}\|_{i}, \forall \boldsymbol{v} \in \boldsymbol{H}^{1}(\Omega), i=0,1
$$

where $P$ is the projection in $\boldsymbol{L}^{2}(\Omega)$ onto $\boldsymbol{H}$ defined in (1.5).

We will only prove a stability result and leave the other details to the interested reader. Consider the equivalent formulation (1.6)-(1.7). Taking the inner product of (1.6) with $k\left(\tilde{\boldsymbol{u}}^{n+1}+P \tilde{\boldsymbol{u}}^{n}\right)$ and of (1.7) with $\frac{k}{2}\left(3 p^{n}-p^{n-1}\right)$, since $\nabla P \tilde{\boldsymbol{u}}^{n}=0$, we obtain

$$
\begin{aligned}
\left(\tilde{\boldsymbol{u}}^{n+1}-\tilde{\boldsymbol{u}}^{n}, \tilde{\boldsymbol{u}}^{n+1}+P \tilde{\boldsymbol{u}}^{n}\right) & +\frac{\nu k}{2}\left\|\nabla\left(\tilde{\boldsymbol{u}}^{n+1}+P \tilde{\boldsymbol{u}}^{n}\right)\right\|^{2} \\
& +\frac{k^{2}}{4}\left(\nabla\left(p^{n+1}-p^{n}\right), \nabla\left(3 p^{n}-p^{n-1}\right)\right) \\
& =k\left\langle\tilde{\boldsymbol{u}}^{n+1}+P \tilde{\boldsymbol{u}}^{n}, \boldsymbol{f}\left(t_{n+\frac{1}{2}}\right)\right\rangle \\
& \leq \frac{\nu k}{4}\left\|\nabla\left(\tilde{\boldsymbol{u}}^{n+1}+P \tilde{\boldsymbol{u}}^{n}\right)\right\|^{2}+C k\left\|\boldsymbol{f}\left(t_{n+\frac{1}{2}}\right)\right\|_{-1}^{2} .
\end{aligned}
$$

We derive from (1.4) and (1.7) that

$$
\begin{aligned}
& P \tilde{\boldsymbol{u}}^{n}-\tilde{\boldsymbol{u}}^{n}=-\frac{1}{2} k \nabla\left(p^{n}-p^{n-1}\right), \\
& \left(\tilde{\boldsymbol{u}}^{n+1}-\tilde{\boldsymbol{u}}^{n}, \nabla \gamma\right)-\frac{1}{2} k\left(\nabla\left(p^{n+1}-2 p^{n}+p^{n-1}\right), \nabla \gamma\right)=0, \forall \gamma \in H^{1}(\Omega) / \mathbb{R} .
\end{aligned}
$$

Therefore,

$$
\begin{aligned}
\left(\tilde{\boldsymbol{u}}^{n+1}-\tilde{\boldsymbol{u}}^{n}, \tilde{\boldsymbol{u}}^{n+1}+P \tilde{\boldsymbol{u}}^{n}\right)=\left\|\tilde{\boldsymbol{u}}^{n+1}\right\|^{2}-\left\|\tilde{\boldsymbol{u}}^{n}\right\|^{2}+\left(\tilde{\boldsymbol{u}}^{n+1}-\tilde{\boldsymbol{u}}^{n}, P \tilde{\boldsymbol{u}}^{n}-\tilde{\boldsymbol{u}}^{n}\right) \\
=\left\|\tilde{\boldsymbol{u}}^{n+1}\right\|^{2}-\left\|\tilde{\boldsymbol{u}}^{n}\right\|^{2}-\frac{1}{2} k\left(\tilde{\boldsymbol{u}}^{n+1}-\tilde{\boldsymbol{u}}^{n}, \nabla\left(p^{n}-p^{n-1}\right)\right) \\
=\left\|\tilde{\boldsymbol{u}}^{n+1}\right\|^{2}-\left\|\tilde{\boldsymbol{u}}^{n}\right\|^{2}-\frac{1}{4} k^{2}\left(\nabla\left(p^{n+1}-2 p^{n}+p^{n-1}\right), \nabla\left(p^{n}-p^{n-1}\right)\right) \\
=\left\|\tilde{\boldsymbol{u}}^{n+1}\right\|^{2}-\left\|\tilde{\boldsymbol{u}}^{n}\right\|^{2}-\frac{k^{2}}{8}\left(\left\|\nabla\left(p^{n+1}-p^{n}\right)\right\|^{2}-\left\|\nabla\left(p^{n}-p^{n-1}\right)\right\|^{2}\right) \\
\quad+\frac{k^{2}}{8}\left\|\nabla\left(p^{n+1}-2 p^{n}+p^{n-1}\right)\right\|^{2} .
\end{aligned}
$$


On the other hand,

$$
\begin{aligned}
\frac{k^{2}}{4}\left(\nabla\left(p^{n+1}-p^{n}\right),\right. & \left.\nabla\left(3 p^{n}-p^{n-1}\right)\right) \\
= & \frac{k^{2}}{4}\left(\left\|\nabla p^{n+1}\right\|^{2}-\left\|\nabla p^{n}\right\|^{2}-\left\|\nabla\left(p^{n+1}-p^{n}\right)\right\|^{2}\right) \\
& +\frac{k^{2}}{4}\left(\nabla\left(p^{n+1}-p^{n}\right), \nabla\left(p^{n}-p^{n-1}\right)\right) \\
= & -\frac{k^{2}}{8}\left\|\nabla\left(p^{n+1}-2 p^{n}+p^{n-1}\right)\right\|^{2} \\
& +\frac{k^{2}}{4}\left(\left\|\nabla p^{n+1}\right\|^{2}-\left\|\nabla p^{n}\right\|^{2}-\left\|\nabla\left(p^{n+1}-p^{n}\right)\right\|^{2}\right) \\
& \quad+\frac{k^{2}}{8}\left(\left\|\nabla\left(p^{n+1}-p^{n}\right)\right\|^{2}+\left\|\nabla\left(p^{n}-p^{n-1}\right)\right\|^{2}\right) .
\end{aligned}
$$

We derive from (1.7) that

$$
\frac{k^{2}}{4}\left\|\nabla\left(p^{m+1}-p^{m}\right)\right\|^{2} \leq\left\|\tilde{\boldsymbol{u}}^{m+1}\right\|^{2} .
$$

Summing up (4.4) for $n=1, m$, and collecting the above inequalities, we arrive at

$$
\frac{\nu k}{4} \sum_{n=1}^{m}\left\|\nabla\left(\tilde{\boldsymbol{u}}^{n+1}+P \tilde{\boldsymbol{u}}^{n}\right)\right\|^{2}+\frac{k^{2}}{8}\left\|\nabla p^{m+1}\right\|^{2} \leq\left\|\boldsymbol{u}^{1}\right\|^{2}+\frac{k^{2}}{8}\left\|\nabla p^{1}\right\|^{2}+\|\boldsymbol{f}\|_{C\left([0, T] ; \boldsymbol{H}^{-1}\right)}^{2} .
$$

Hence, the scheme is stable.

\section{NUMERICAL RESUlTS}

We now present some numerical results by using the schemes (1.3)-(1.4) and (1.10)-(1.11). Since the dominating error in these schemes is introduced by the approximation of the linear operator, we shall only perform numerical tests on the Navier-Stokes equations linearized at $\boldsymbol{u}=0$.

Let $\Omega=(-1,1)^{2}, \nu=1$ and the exact solution $(\boldsymbol{u}, p)$ of the linearized (at $\boldsymbol{u}=0$ ) Navier-Stokes equations to be

$$
\begin{aligned}
\boldsymbol{u}(x, y, t) & =\pi \log (1+t)\left(\sin 2 \pi y \sin ^{2} \pi x,-\sin 2 \pi x \sin ^{2} \pi y\right), \\
p(x, y, t) & =\log (1+t) \cos \pi x \sin \pi y .
\end{aligned}
$$

Then the function $\boldsymbol{f}$ is given by $\boldsymbol{f}=\boldsymbol{u}_{t}-\Delta \boldsymbol{u}+\nabla p$. All computations are started from the initial time $t_{0}=0$.

For the space discretization, we use the Legendre-Galerkin method (cf. [19]) with 33 modes in each direction for the velocity and the pressure. Thanks to the high accuracy of the spectral method, the error introduced by the space discretization is negligible compared to the error introduced by the time discretization for this specific example. Denote

$$
\operatorname{err}_{u}(t, k)=\frac{\max _{i, j=0, \ldots, 32}\left|\boldsymbol{u}\left(x_{i}, y_{j}, t\right)-\boldsymbol{u}^{\frac{t}{k}}\left(x_{i}, y_{j}\right)\right|}{\max _{i, j=0, \ldots, 32}\left|\boldsymbol{u}\left(x_{i}, y_{j}, t\right)\right|},
$$




$$
\operatorname{err}_{p}(t, k)=\frac{\sqrt{\sum_{i, j=0}^{32}\left|p\left(x_{i}, y_{j}, t\right)-p^{\frac{t}{k}}\left(x_{i}, y_{j}\right)\right|^{2}}}{33 \max _{i, j=0, \ldots, 32}\left|p\left(x_{i}, y_{j}, t\right)\right|},
$$

where $\left(x_{i}, y_{j}\right)=\left(\cos \frac{i \pi}{32}, \cos \frac{j \pi}{32}\right)(i, j=0, \ldots, 32)$ are the Gauss-Lobatto collocation points. In order to determine the numerical convergence rate, we set

$$
\mathrm{r}_{u}(t, k)=\frac{\operatorname{err}_{u}(t, k)}{\operatorname{err}_{u}(t, k / 2)}, \quad \mathrm{r}_{p}(t, k)=\frac{\operatorname{err}_{p}(t, k)}{\operatorname{err}_{p}(t, k / 2)} .
$$

We use the $l^{2}$-norm to measure the error of the pressure approximations because the pressure approximations exhibit large error near the boundary, so that the $l^{\infty}$-norm of the error does not properly represent the errors in the interior of the domain. On the other hand, the $l^{2}$-norm and $l^{\infty}$-norm of the error for the velocity approximations appear to behave similarly, at least for this specific example. Hence, the $l^{\infty}$-norm is used to measure the error of the velocity approximations.

TABLE I. Error behavior of the scheme (1.10)-(1.11) with $\beta=\frac{1}{4}$

\begin{tabular}{|c|cc|cc|cc|cc|}
\hline$k$ & $\operatorname{err}_{u}(1, k)$, & $\mathrm{r}_{u}(1, k)$ & $\operatorname{err}_{p}(1, k)$, & $\mathrm{r}_{p}(1, k)$ & $\operatorname{err}_{u}(5, k)$, & $\mathrm{r}_{u}(5, k)$ & $\operatorname{err}_{p}(5, k)$, & $\mathrm{r}_{p}(5, k)$ \\
\hline 0.1 & $2.43 \mathrm{E}-3$, & 4.13 & $7.39 \mathrm{E}-2$, & 2.25 & $3.52 \mathrm{E}-4$, & 4.04 & $2.43 \mathrm{E}-2$, & 2.41 \\
& $(2.06 \mathrm{E}-3)$, & $(4.32)$ & & & $(2.95 \mathrm{E}-4)$, & $(4.09)$ & & \\
\hline 0.05 & $5.89 \mathrm{E}-4$, & 4.03 & $3.29 \mathrm{E}-2$, & 2.33 & $8.71 \mathrm{E}-5$, & 4.44 & $1.01 \mathrm{E}-2$, & 2.36 \\
& $(4.76 \mathrm{E}-4)$, & $(3.90)$ & & & $(7.21 \mathrm{E}-5)$, & $(4.01)$ & & \\
\hline 0.025 & $1.46 \mathrm{E}-4$, & 4.02 & $1.41 \mathrm{E}-2$, & 2.33 & $1.96 \mathrm{E}-5$, & 3.78 & $4.28 \mathrm{E}-3$, & 1.88 \\
& $(1.22 \mathrm{E}-4)$, & $(4.01)$ & & & $(1.80 \mathrm{E}-5)$, & $(4.35)$ & & \\
\hline 0.0125 & $3.63 \mathrm{E}-5$ & & $6.06 \mathrm{E}-3$ & & $5.19 \mathrm{E}-6$ & & $2.28 \mathrm{E}-3$ & \\
& $(3.04 \mathrm{E}-5)$ & & & & $(4.14 \mathrm{E}-6)$ & & & \\
\hline
\end{tabular}

$\left({ }^{*}\right)$ the results in parentheses are for the error $u(t)-P u^{\frac{t}{k}}$.

TABLE II. Error behavior of the scheme (1.3)-(1.4)

\begin{tabular}{|c|cc|cc|cc|cc|}
\hline$k$ & $\operatorname{err}_{u}(1, k)$, & $\mathrm{r}_{u}(1, k)$ & $\operatorname{err}_{p}(1, k)$, & $\mathrm{r}_{p}(1, k)$ & $\operatorname{err}_{u}(5, k)$, & $\mathrm{r}_{u}(5, k)$ & $\operatorname{err}_{p}(5, k)$, & $\mathrm{r}_{p}(5, k)$ \\
\hline 0.1 & $2.06 \mathrm{E}-3$, & 4.32 & $7.64 \mathrm{E}-2$, & 2.31 & $2.95 \mathrm{E}-4$, & 4.09 & $2.45 \mathrm{E}-2$, & 2.43 \\
\hline 0.05 & $4.76 \mathrm{E}-4$, & 3.90 & $3.31 \mathrm{E}-2$, & 2.40 & $7.21 \mathrm{E}-5$, & 4.01 & $1.01 \mathrm{E}-2$, & 2.37 \\
\hline 0.025 & $1.22 \mathrm{E}-4$, & 4.01 & $1.38 \mathrm{E}-2$, & 2.39 & $1.80 \mathrm{E}-5$, & 4.35 & $4.27 \mathrm{E}-3$, & 1.88 \\
\hline 0.0125 & $3.04 \mathrm{E}-5$ & & $5.78 \mathrm{E}-3$ & & $4.14 \mathrm{E}-6$ & & $2.27 \mathrm{E}-3$ & \\
\hline
\end{tabular}

In Tables I and II, we list the errors of the velocity approximations and of the pressure approximations at $t=1$ and $t=5$ by using the schemes (1.10)-(1.11) and (1.3)-(1.4) respectively. For the scheme (1.10)-(1.11), we have also computed the error between $\boldsymbol{u}(t)$ and $P \boldsymbol{u}^{\frac{t}{k}}$. The latter is the projection of $\boldsymbol{u}^{\frac{t}{k}}$ onto the divergence-free space $\boldsymbol{H}$. We note that this projection is only performed at the last time step, not at every time step as in (1.3)-(1.4).

The results in Tables I and II clearly indicate that the two schemes are secondorder accurate for the velocity and at least first-order accurate (in the $l^{2}$-norm) for the pressure. In Figure 1, we plot the pressure error for the two schemes at $t=1$ with three different time steps. It is clear that both pressure approximations exhibit a numerical boundary layer whose width decreases as the time step decreases. It is also clear that the numerical boundary layers are consequences of the incompatible homogeneous Neumann boundary condition for the pressure approximation, since 

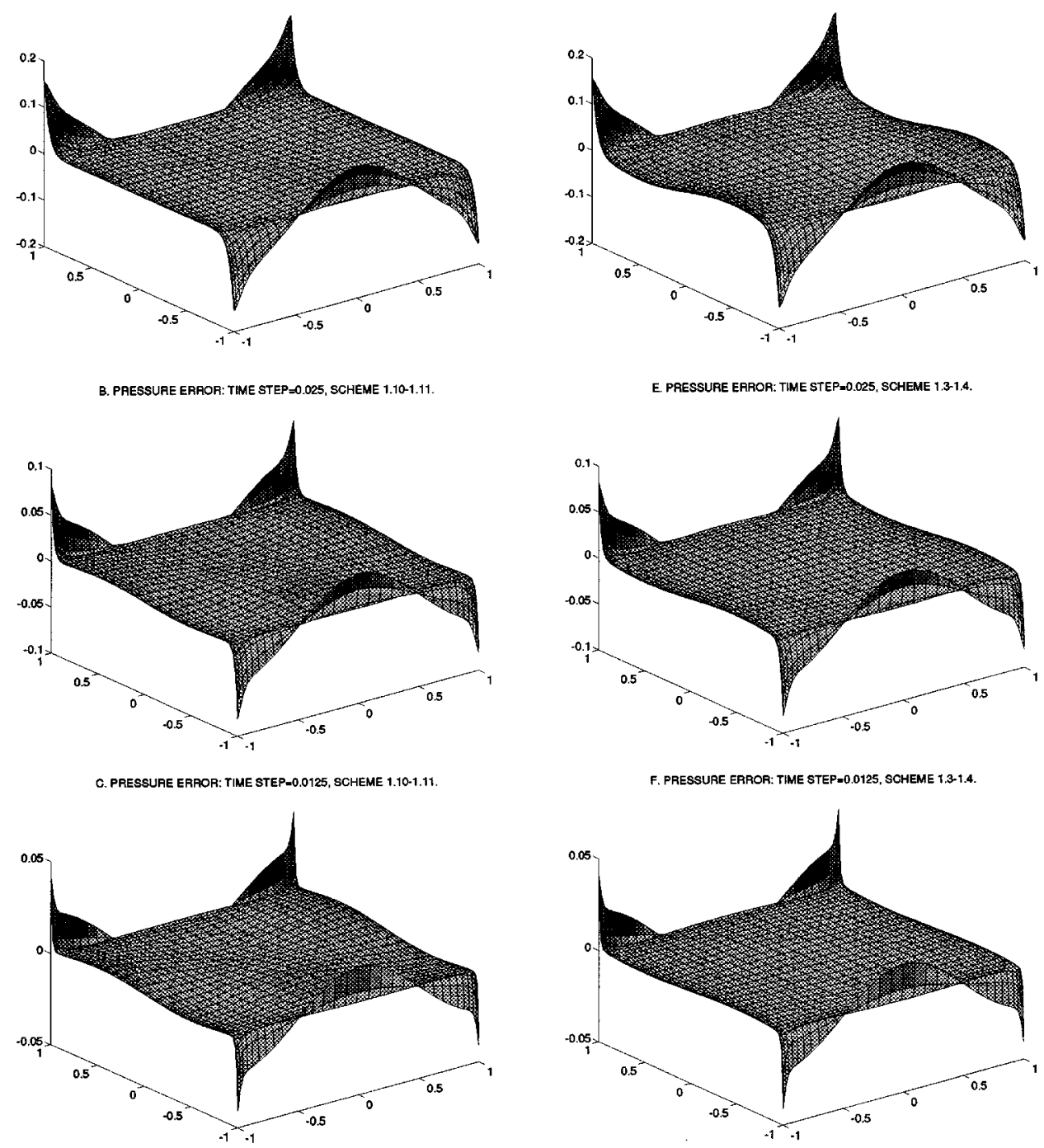

Figure 1. Plots of pressure errors at $t=1$

the numerical boundary layers only appear at the two boundaries $\{(x, y): x \in$ $(-1,1), y= \pm 1\}$ where the exact pressure is such that $\frac{\partial p}{\partial \vec{n}} \neq 0\left(\frac{\partial p}{\partial \vec{n}}=0\right.$ at the other two boundaries). On the other hand, there is no numerical boundary layer for the velocity approximations (see Figure 2).

In order to determine the accuracy of the pressure approximations away from the numerical boundary layer, we set

$$
\operatorname{err}_{p}^{1}(t, k)=\frac{\sqrt{\sum_{i, j=3}^{29}\left|p\left(x_{i}, y_{j}, t\right)-p^{\frac{t}{k}}\left(x_{i}, y_{j}\right)\right|^{2}}}{27 \max _{i, j=3, \ldots, 29}\left|p\left(x_{i}, y_{j}, t\right)\right|}, \mathbf{r}_{p}^{1}(t, k)=\frac{\operatorname{err}_{p}^{1}(t, k)}{\operatorname{err}_{p}^{1}(t, k / 2)},
$$

which represents the error in the $l^{2}$-norm of the pressure approximations away from the numerical boundary layer. The corresponding results are tabulated in Table 

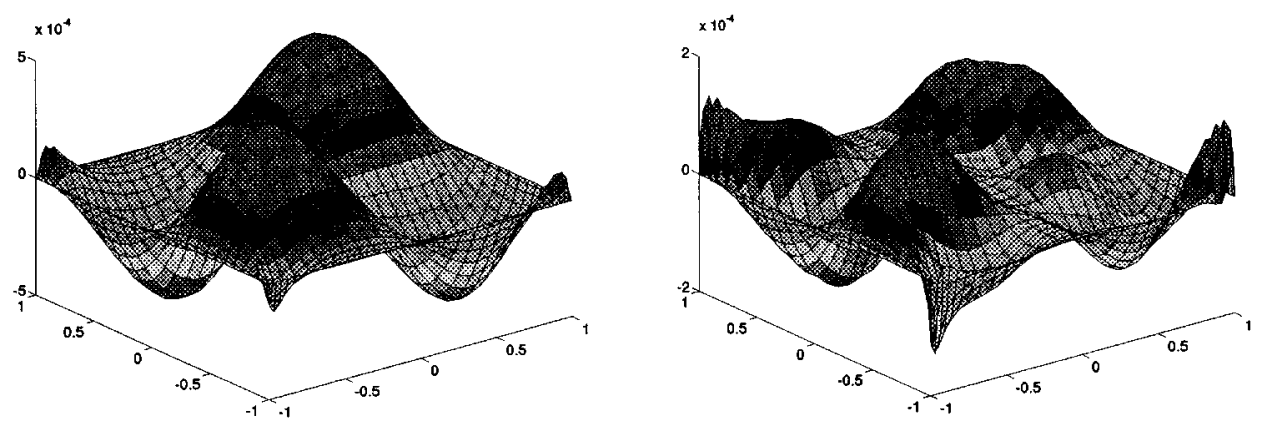

Figure 2. Plots of (first-component) velocity errors at $t=1$

III. We notice that away from the boundary layer, the scheme (1.3)-(1.4) seems to be second-order accurate for the pressure, while the scheme (1.10)-(1.11) remains first-order accurate because the truncation error for the pressure is only first-order accurate.

TABLE III. Error behavior of the pressure approximations in the interior of $\Omega$

\begin{tabular}{|c|cc|cc|}
\hline & Scheme & $(1.10)-(1.11)$ & Scheme & $(1.3)-(1.4)$ \\
\hline$k$ & $\operatorname{err}_{p}^{1}(1, k)$, & $\mathrm{r}_{p}^{1}(1, k)$ & $\operatorname{err}_{p}^{1}(1, k)$, & $\mathrm{r}_{p}^{1}(1, k)$ \\
\hline 0.1 & $2.54 \mathrm{E}-2$, & 3.87 & $3.36 \mathrm{E}-2$, & 4.30 \\
\hline 0.05 & $6.57 \mathrm{E}-3$, & 2.00 & $7.81 \mathrm{E}-3$, & 4.49 \\
\hline 0.025 & $3.28 \mathrm{E}-3$, & 1.72 & $1.74 \mathrm{E}-3$, & 3.88 \\
\hline 0.0125 & $1.91 \mathrm{E}-3$ & & $4.48 \mathrm{E}-4$ & \\
\hline
\end{tabular}

The convergence rate of the scheme (1.10)-(1.11) is insensitive to the choice of $\beta$, as long as $\beta \geq \frac{1}{4}$, although larger $\beta$ introduce larger truncation errors and give less accurate results. Therefore, it is recommended to choose $\beta=\frac{1}{4}$ for (1.10)-(1.11).

Finally, we note that the results by (1.10)-(1.11) and (1.3)-(1.4) are of comparable accuracy. In fact, $\boldsymbol{u}^{\frac{t}{k}}$ in (1.10)-(1.11) is slightly less accurate than $\boldsymbol{u}^{\frac{t}{k}}$ in (1.3)-(1.4). But it is interesting to note (cf. Tables II and III) that for this example, the error between $\boldsymbol{u}(t)$ and $P \boldsymbol{u}^{\frac{t}{k}}$ in (1.10)-(1.11) is numerically identical to the error between $\boldsymbol{u}(t)$ and $\boldsymbol{u}^{\frac{t}{k}}$ in (1.3)-(1.4).

\section{APPENDIX}

Lemma A1. Let $\boldsymbol{R}^{n}, \boldsymbol{E}_{r}^{n}$ and $E_{p}^{n}$ be defined respectively in (2.21) and (2.29). We assume (2.13) and (2.15). Then there exist $c_{1}, c_{2}, c_{3}>0$ such that

$$
\begin{array}{r}
k \sum_{n=1}^{M-1}\left\|\boldsymbol{R}_{1}^{n}\right\|_{-1}^{2} \leq c_{1} k^{4} \int_{t_{0}}^{T_{1}}\left(\left\|\boldsymbol{u}_{t t t}(s)\right\|_{-1}^{2}+\left\|\boldsymbol{u}_{t t}(s)\right\|_{1}^{2}+\left\|p_{t t}(s)\right\|^{2}\right) d s, \\
\left\|\boldsymbol{R}^{n}\right\| \leq c_{2} k\left(\max _{t \in\left[t_{0}, T_{1}\right]}\left\|\boldsymbol{u}_{t t}(t)\right\|+\max _{t \in\left[t_{0}, T_{1}\right]}\left\|\boldsymbol{u}_{t}(t)\right\|_{2}+\max _{t \in\left[t_{0}, T_{1}\right]}\left\|p_{t}(t)\right\|_{1}\right), \\
\forall 0 \leq n \leq M-1,
\end{array}
$$

$$
k \sum_{n=2}^{M-1}\left\|E_{p}^{n}\right\|_{1}^{2} \leq c_{3} k^{4} \int_{t_{0}}^{T_{1}}\left\|p_{t t}(s)\right\|_{1}^{2} d s .
$$


Proof. A result similar to (A.1) was proved in Lemma 1 of [17]. It is clear that the argument in $[\mathbf{1 7}]$ can be directly used to prove (A.1). One can also easily derive (A.2) by using similar arguments.

By using Taylor's Theorem with remainder in integral form, we have

$$
\begin{aligned}
& p\left(t_{n+1}\right)=p\left(t_{n}\right)+k p_{t}\left(t_{n}\right)+\int_{t_{n}}^{t_{n+1}} p_{t t}(s)\left(t_{n+1}-s\right) d s, \\
& p\left(t_{n-2}\right)=p\left(t_{n-1}\right)-k p_{t}\left(t_{n-1}\right)+\int_{t_{n-2}}^{t_{n-1}} p_{t t}(s)\left(s-t_{n-2}\right) d s .
\end{aligned}
$$

We derive from the above that

$$
\begin{aligned}
E_{p}^{n} & =\left(p\left(t_{n+1}\right)-p\left(t_{n}\right)\right)-\left(p\left(t_{n-1}\right)-p\left(t_{n-2}\right)\right) \\
& =k \int_{t_{n-1}}^{t_{n}} p_{t t}(s) d s+\int_{t_{n}}^{t_{n+1}} p_{t t}(s)\left(t_{n+1}-s\right) d s+\int_{t_{n-2}}^{t_{n-1}} p_{t t}(s)\left(s-t_{n-2}\right) d s .
\end{aligned}
$$

By using the Schwarz inequality, we derive from the above that

$$
\left\|\nabla E_{p}^{n}\right\|^{2} \leq k^{3} \int_{t_{n-1}}^{t_{n}}\left\|\nabla p_{t t}(s)\right\|^{2} d s+\frac{1}{3} k^{3}\left(\int_{t_{n-2}}^{t_{n-1}}+\int_{t_{n}}^{t_{n+1}}\right)\left\|\nabla p_{t t}(s)\right\|^{2} d s .
$$

We conclude that

$$
k \sum_{n=2}^{M-1}\left\|\nabla E_{p}^{n}\right\|^{2} \leq \frac{5}{3} k^{4} \int_{t_{0}}^{T_{1}}\left\|\nabla p_{t t}(s)\right\|^{2} d s .
$$

Lemma A2. We assume (2.13), (2.15), (2.12) and $\beta>\frac{1}{4}$. Then for any fixed integer $m$, there exists a positive constant $c_{4}$ depending on the data and $m$ such that

$$
\left\|\boldsymbol{u}\left(t_{i}\right)-\boldsymbol{u}^{i}\right\|^{2}+k^{2}\left\|\nabla\left(p\left(t_{i}\right)-p^{i}\right)\right\|^{2} \leq c_{4} k^{4}, \quad i=1, \ldots, m,
$$

where $\left(\boldsymbol{u}^{i}, p^{i}\right)$ is the solution of (1.10)-(1.11).

Proof. Let $\delta=\beta-\frac{1}{4}>0$. Taking the inner product of (2.17) at $n=0$ with $k\left(\boldsymbol{e}^{1}+\boldsymbol{e}^{0}\right)$ and of (2.18) at $n=0$ with $k q^{0}$, and summing up the two relations, we get

$$
\begin{aligned}
\left\|\boldsymbol{e}^{1}\right\|^{2}- & \left\|\boldsymbol{e}^{0}\right\|^{2}+\frac{\nu k}{2}\left\|\nabla\left(\boldsymbol{e}^{1}+\boldsymbol{e}^{0}\right)\right\|^{2}+\frac{\beta k^{2}}{2}\left\{\left\|\nabla q^{1}\right\|^{2}-\left\|\nabla q^{0}\right\|^{2}-\left\|\nabla\left(q^{1}-q^{0}\right)\right\|^{2}\right\} \\
= & k\left(\nabla \boldsymbol{e}^{0}, q^{0}\right)+\beta k^{2}\left(\nabla\left(p\left(t_{1}\right)-p\left(t_{0}\right)\right), \nabla q^{0}\right)+k\left(\boldsymbol{R}^{0}+\boldsymbol{Q}^{0}, \boldsymbol{e}^{1}+\boldsymbol{e}^{0}\right) \\
& \quad(\operatorname{using}(2.20) \text { and }(2.3)) \\
\leq & C\left\|\boldsymbol{e}^{0}\right\|^{2}+C k^{2}\left(\left\|\nabla q^{0}\right\|^{2}+\left\|\nabla\left(p\left(t_{1}\right)-p\left(t_{0}\right)\right)\right\|^{2}\right) \\
& \quad+k\left(\boldsymbol{R}^{0}, \boldsymbol{e}^{1}+\boldsymbol{e}^{0}\right)-k \tilde{b}\left(\tilde{\boldsymbol{e}}^{\frac{1}{2}}, \tilde{\boldsymbol{u}}\left(t_{\frac{1}{2}}\right), \boldsymbol{e}^{1}+\boldsymbol{e}^{0}\right) \\
\leq & \frac{\delta}{4(\delta+1)}\left\|\boldsymbol{e}^{1}\right\|^{2}+C\left\|\boldsymbol{e}^{0}\right\|^{2} \\
& \quad+C k^{2}\left(\left\|\nabla q^{0}\right\|^{2}+\left\|\nabla\left(p\left(t_{1}\right)-p\left(t_{0}\right)\right)\right\|^{2}+\left\|\boldsymbol{R}^{0}\right\|^{2}+\left\|\nabla\left(\boldsymbol{e}^{1}+\boldsymbol{e}^{0}\right)\right\|^{2}\right) .
\end{aligned}
$$


On the other hand, the relation $(2.25)$ at $m=0$ reads

$$
\frac{\beta k^{2}}{2}\left\|\nabla\left(q^{1}-q^{0}\right)\right\|^{2} \leq \frac{1+\frac{\delta}{2}}{1+\delta}\left\|\boldsymbol{e}^{1}\right\|^{2}+C k^{4}+\frac{\left(1-\frac{\delta}{2}\right) \beta k^{2}}{2}\left(\left\|\nabla q^{1}\right\|^{2}+\left\|\nabla q^{0}\right\|^{2}\right) .
$$

We then derive from the last two inequalities, (2.12) and Lemma A1 that for $k$ sufficiently small

$$
\frac{\delta}{4(1+\delta)}\left\|\boldsymbol{e}^{1}\right\|^{2}+\frac{\nu k}{2}\left\|\nabla\left(\boldsymbol{e}^{1}+\boldsymbol{e}^{0}\right)\right\|^{2}+\frac{\delta \beta k^{2}}{4}\left\|\nabla q^{1}\right\|^{2} \leq C k^{4} .
$$

We can conclude by repeating the above process $m-1$ times.

\section{REFERENCES}

1. J. Bell, P. Colella and H. Glaz, A second-order projection method for the ioncompressible Navier-Stokes equations, J. Comput. Phys. 85 (1989), 257-283. MR 90i:76002

2. A. J. Chorin, On the convergence of discrete approximations to the Navier-Stokes equations, Math. Comp. 23 (1969), 341-353. MR 39:3724

3. M. Deville, L. Kleiser and F. Montigny-Rannou, Pressure and time treatment for Chebyshev spectral solution of a Stokes problem, Intern. J. Numer. Methods in Fluids 4 (1984), 11491163.

4. W. E. and J. G. Liu, Projection method I: Convergence and numerical boundary layers, SIAM J. Numer Anal. 32 (1995), 1017-1057. CMP 95:15

5. P. M. Gresho, On the theory of semi-implicit projection methods for viscous incompressible flow and its implementation via a finite element method that also introduces a nearly consistent mass matrix. Part I: Theory, Intern. J. Numer. Methods in Fluids 11 (1990), 587-620. MR 91m:76071a

6. P. M. Gresho and R. L. Sani, On pressure boundary conditions for the incompressible NavierStokes equations, Intern. J. Numer. Methods in Fluids 7 (1987), 1111.

7. J. G. Heywood and R. Rannacher, Finite element apoproximation of the nonstationary NavierStokes problem. I. Regularity of solutions and second-order error estimates for spatial discretization, SIAM J. Numer. Anal. 19 (1982), 275-311. MR 83d:65260

8. __ Finite element approximation of the nonstationary Navier-Stokes problem. IV. Error analysis for second-order time discretization, SIAM J. Numer. Anal. 27 (1990), 353-384. MR 92c: 65133

9. G. E. Karniadakis, M. Israeli and S. A. Orszag, High-order splitting methods for the incompressible Navier-Stokes equations, J. Comput. Phys. 97 (1991), 414-443. MR 92h:76066

10. J. Kim and P. Moin, Application of a fractional-step method to incompressible Navier-Stokes equations, J. Comput. Phys. 59 (1985), 308-323. MR 87a:76046

11. S. A. Orszag, M. Israeli and M. Deville, Boundary conditions for incompressible flows, J. Sci. Comput. 1 (1986), 75-111.

12. R. Rannacher, On Chorin's projection method for the incompresible Navier-Stokes Equations, The Navier-Stokes Equations II. Theory and Numerical Methods, Lecture Notes in Mathematics, vol. 1530, 1991, pp. 167-183. MR 95a:65149

13. __ Numerical analysis of the Navier-Stokes equations, Appl. Math., vol. 38, 1993, pp. 361-380. MR 94h:65101

14. J. Shen, A new pseudo-compressibility method for the Navier-Stokes equations, Penn State Math. Dept. Report AM137, 1994, to appear in Appl. Numer. Math..

15. - On error estimates of the projection methods for the Navier-Stokes equations: firstorder schemes, SIAM J. Numer. Anal. 29 (1992), 57-77. MR 92m:35213

16. _ On pressure stabilization method and projection method for unsteady Navier-Stokes equations, Advances in Computer Methods for Partial Differential Equations (R. Vichnevetsky, D. Knight and G. Richter, eds.), IMACS, 1992, pp. 658-662.

17. for Navier-Stokes equations, Numer. Math. 62 (1992), 49-73. MR 93a:35122 
18. _ A remark on the projection-3 methods, Intern. J. Numer. Methods in Fluids 16 (1993), 249-253. MR 93k:76082

19. __ Efficient spectral-Gelerkin method I. Direct solvers for second- and fourth-order equations by using Legendre polynomials, SIAM J. Sci. Comput. 15 (1994), 1489-1505. MR 95j:65150

20. R. Temam, Sur l'approximation de la solution des équations de Navier-Stokes par la méthode des pas fractionnaires II, Arch. Rat. Mech. Anal. 33 (1969), 377-385. MR 39:5968

21. N Navier-Stokes equations and nonlinear functional analysis, CBMS-NSF Regional Conference Series in Applied Mathematics, vol. 41, SIAM, Philadelphia, 1983. MR 86f:35152

22. _ Navier-Stokes Equations: Theory and Numerical Analysis (1984), North-Holland, Amsterdam. MR 58:29439

23. J. Van Kan, A second-order accurate pressure-correction scheme for viscous incompressible flow, SIAM J. Sci. Stat. Comput. 7 (1986), 870-891. MR 87h:76008

Department of Mathematics, Penn State University, University Park, Pennsylvania 16802

Current address: Department of Mathematics, Penn State University, University Park, Pennsylvania 16802

E-mail address: shen_j@math.psu.edu 